Supporting Information (SI) for:

\title{
Ultrafast Triplet State Formation in a Methylated Fungi- Derived Pigment: Towards Rational Molecular Design for Sustainable Optoelectronics
}

Taylor D. Krueger, ${ }^{a}$ Longteng Tang, ${ }^{a}$ Gregory Giesbers, ${ }^{b}$ Ray C. Van Court, ${ }^{\mathrm{c}}$ Liangdong Zhu, ${ }^{\mathrm{a}}$ Seri C. Robinson, ${ }^{\mathrm{c}}$ Oksana Ostroverkhova, ${ }^{\mathrm{b}}$ and Chong Fang, ${ }^{* a \mathrm{a}}$

${ }^{a}$ Department of Chemistry, Oregon State University, 153 Gilbert Hall, Corvallis, Oregon 97331

${ }^{\mathrm{b}}$ Department of Physics, Oregon State University, 301 Weniger Hall, Corvallis, Oregon 97331

${ }^{\mathrm{c}}$ Department of Wood Science and Engineering, Oregon State University, 119 Richardson Hall, Corvallis, Oregon 97331

* To whom correspondence should be addressed. E-mail: Chong.Fang@oregonstate.edu 
SI Text

Experimental Materials and Methods

\section{SI Figures}

Figure S1. Steady-state electronic spectra of xylindein and dimethylxylindein $\quad S 9-S 11$

Figure S2. Steady-state absorption vs. ground state bleaching in TA of dimethylxylindein in various solvents and EAS from global analysis of dimethylxylindein in DCM S12-S14

Figure S3. Fs-TA spectra and global analysis of dimethylxylindein in DCM after $560 \mathrm{~nm}$ excitation

S15-S18

Figure S4. Probe-dependent fs-TA intensity dynamics of dimethylxylindein in DCM after $560 \mathrm{~nm}$ excitation S19-S21

Figure S5. Fs-TA spectra, global analysis, and probe-dependent intensity dynamics of dimethylxylindein in ethanol after $400 \mathrm{~nm}$ excitation S22-S23

Figure S6. Fs-TA spectra, global analysis, and probe-dependent intensity dynamics of dimethylxylindein thin films after $560 \mathrm{~nm}$ excitation S24-S25

Figure S7. Raman marker band intensity dynamics from ES-FSRS of dimethylxylindein in DCM after $560 \mathrm{~nm}$ excitation S26-S27

Figure S8. GS and ES-FSRS spectra of dimethylxylindein and xylindein S28-S29

Figure S9. Calculated vs. experimental Raman spectra of dimethylxylindein in DCM S30

Figure S10. Illustrations of dimethylxylindein 1619 and $1549 \mathrm{~cm}^{-1}$ Raman modes $\quad S 31$ Figure S11. Quantum calculation results of dimethylxylindein with various planarity S32 Additional discussions follow each figure.

\section{SI Table}

Table S1. Vibrational mode assignment for dimethylxylindein in DCM 


\section{$\underline{\text { SI Text }}$}

\section{Experimental Materials and Methods:}

Sample preparation. The synthesis of dimethylxylindein started with the extraction, growth, and purification of xylindein. ${ }^{1-3}$ Xylindein was isolated from decaying hardwood in Haliburton, Ontario, Canada; using established methods, the fungal pigment was extracted from laboratorygrown cultures of Chlorociboria aeruginosa (strain UAMH 11657). ${ }^{1,45}$ Inoculation of $50 \mathrm{~mL}$ of sterile $2 \%$ malt agar liquid media by cultures maintained on $2 \%$ malt agar plates occurred in 250 $\mathrm{mL}$ borosilicate mason jars. Growth proceeded in the jars sealed with Parafilm M at ambient conditions $\left(21{ }^{\circ} \mathrm{C}\right.$ and $35 \%$ relative humidity) for three months with shaking at $99 \mathrm{rpm}$ (Orbital Gene Scientific Industries, Bohemia, NY, USA). After growth, the fungal colonies were broken up by blending the liquid media for two minutes (Oster classic series blender, Atlanta, GA, USA). For every $50 \mathrm{~mL}$ of liquid media, $30 \mathrm{~mL}$ of dichloromethane (DCM, HPLC grade; VWR, Radnor, PA) was added in a $500 \mathrm{~mL}$ Erlenmeyer flask and manually swirled for three minutes to mix the water and DCM layers. Extraction of xylindein from the DCM layer went through a $250 \mathrm{~mL}$ separatory funnel before evaporation of DCM. To remove most contaminants detrimental to the conductivity of xylindein thin films, dry xylindein was rinsed several times with ethanol. ${ }^{2}$

To synthesize dimethylxylindein, the methylating agent $\mathrm{TMSCHN}_{2}(2.0 \mathrm{M}$ in hexane, 2.64 $\mathrm{mL}$ ) was added to $150 \mathrm{mg}$ of xylindein in $\mathrm{CHCl}_{3}: \mathrm{MeOH}(20 \mathrm{~mL}: 5 \mathrm{~mL})$ mixed solvents at room temperature. The solution color changed from dark green to dark purple after three hours, which indicated conversion of xylindein to dimethylxylindein. After the crude mixture was concentrated, purification via silica gel chromatography (20:1 DCM:acetone) yielded $75 \mathrm{mg}$ magenta amorphous solid. Spectral data matching those previously reported ${ }^{4}$ confirmed the successful production of dimethylxylindein (99\% purity, NMR analysis). Thin films of dimethylxylindein were prepared by repeated spin-casting onto glass substrates at $1,000 \mathrm{rpm}$, reaching a final thickness of $3-5 \mu \mathrm{m}$., 
Steady-state spectroscopic measurements. Xylindein and dimethylxylindein were used as is after the initial extraction and purification of the fungal pigment for steady-state and the ensuing timeresolved spectroscopic measurements. When necessary, dimethylxylindein was diluted with the lab-grade solvent DCM (TCI, 99\% purity) or ethanol (Koptec). A Thermo Scientific Evolution $201 \mathrm{UV} /$ Visible (UV/Vis) spectrophotometer measured steady-state absorption spectra of the sample solution housed in a 1-mm-pathlength quartz cuvette (Spectrosil 1-Q-1, Starna Cells). The fluorescence measurements were performed with a Shimadzu RF-6000 spectrofluorophotometer on sample solution housed in a 5-mm-pathlength four-sided rectangular quartz cuvette. ${ }^{6}$

\section{Femtosecond transient absorption (fs-TA) and femtosecond stimulated Raman spectroscopy}

(FSRS). A detailed description of the femtosecond (fs) laser system used for all the ultrafast spectroscopies on dimethylxylindein and xylindein has been reported previously. ${ }^{6-8}$ In brief, a regenerative amplifier (Legend Elite-USP-1K-HE, Coherent, Inc.) seeded by a mode-locked Ti:Sapphire oscillator (Mantis-5, Coherent, Inc.) generates the $\sim 35$ fs pulse train centered around $800 \mathrm{~nm}$ with a $1 \mathrm{kHz}$ repetition rate at $\sim 3.7 \mathrm{~W}$ average power. A home-built two-stage noncollinear optical parametric amplifier (NOPA) allows for a wavelength-tunable actinic pump to excite the desired chromophore, with a neutral density filter significantly reducing the actinic pump power to $\sim 0.25 \mathrm{~mW}$ at $560 \mathrm{~nm}$ for fs-TA and excited-state (ES)-FSRS experiments. An optical chopper synchronized to half of laser repetition rate $(500 \mathrm{~Hz})$ in the actinic pump beampath enables efficient data acquisition (pump on vs. off). Temporal compression of the tunable actinic pulses to $<100$ fs pulse duration was achieved through a chirped mirror pair (DCM-12, 400-700 nm, Laser Quantum, Inc.). ${ }^{9}$ Broadband supercontinuum white light as probe pulse was produced by focusing a small portion of the $800 \mathrm{~nm}$ fundamental pulse onto a 2-mm-pathlength quartz cuvette filled with 
deionized water, then collimated and filtered if necessary. For fs-TA experiments, the probe photons span most of the visible spectrum $(\sim 450-710 \mathrm{~nm})$. The probe was compressed and overlapped with the actinic pump on sample solution housed in a 1-mm-pathlength quartz cuvette with a magnetic stir bar to ensure fresh sample was repeatedly irradiated throughout the data scan.

For ground state (GS) and ES-FSRS measurements, a narrowband ps Raman pump was generated by a home-built three-stage NOPA. A small portion of the tunable fs seed was converted to a ps seed by dispersing the seed with a reflective grating onto a narrow slit to spectrally filter the desired wavelength. This conversion is quite inefficient; ${ }^{10}$ hence, two ps NOPAs following the spectral filter are necessary to obtain sufficient power for FSRS. The tunability of the Raman pump is attainable by altering the phase-matching conditions to achieve the $600,650,675$, and $710 \mathrm{~nm}$ pump wavelengths to resonantly enhance the GS or ES species of interest. ${ }^{8}$ A neutral density filter reduced the Raman pump power at 600 and $710 \mathrm{~nm}$ to 1.5 and $0.7 \mathrm{~mW}$, respectively, after the chopper. For ES-FSRS measurements, the chopper was placed in the Raman pump beampath while an electronic shutter was used for the actinic pump. The fs actinic pump and white-light probe for FSRS measurements were generated similarly as described for fs-TA measurements. ${ }^{8}$

For GS/ES-FSRS and fs-TA, the transmitted probe was directed into an IsoPlane SCT-320 imaging spectrograph (Princeton Instruments, Inc.) and dispersed onto a PIXIS:100F CCD array camera (Princeton Instruments, Inc.) for signal detection. For dispersion, a 1200 groves $/ \mathrm{mm}, 500$ nm blaze wavelength reflective grating was used for FSRS measurements (to achieve high spectral resolution) while a 300 groves $/ \mathrm{mm}, 300 \mathrm{~nm}$ blaze wavelength reflective grating was used for fsTA (to achieve wide spectral coverage). For fs-TA measurements on dimethylxylindein and xylindein in solution (DCM with decent chromophore solubility), the optical density (OD) ranged from $0.4-0.6 / \mathrm{mm}$, primarily to ensure the monomeric chromophores were photoexcited. Due to 
the relative poor solubility of dimethylxylindein in ethanol, we sonicated the solution for $\sim 1$ hour to reach an $\mathrm{OD} \approx 0.4 / \mathrm{mm}$ (see fs-TA spectra in Figure S5 below). At the lowest OD (0.4), we estimated the solute concentration to be $0.27 \mathrm{mM}$, below the typical limit $(\sim 1 \mathrm{mM})$ for intermolecular interactions to occur (e.g., forming aggregates). ${ }^{11}$ For FSRS measurements, the sample OD was $\sim 1.0 / \mathrm{mm}$ and $0.5 / \mathrm{mm}$ for dimethylxylindein and xylindein, respectively, to achieve sufficient signal intensity of vibrational features. ${ }^{8,12}$ The UV-Vis spectra were collected before and after each ultrafast dataset collection to confirm the sample integrity. ${ }^{8,13}$

For fs-TA measurements with an extended time window, a different segment of the optical table was used. Without a tunable setup, a fraction of the $800 \mathrm{~nm}$ fundamental pulse was focused onto a $\mathrm{BBO}$ thin crystal to produce $400 \mathrm{~nm}$ actinic pump via second harmonic generation before reducing the pump power to $\sim 0.2 \mathrm{~mW}$. A quadruple-pass setup (with two UBBR2.5-2S broadband hollow retroreflectors, Newport, Inc.) lengthened the delay line distance (NRT150, Thorlabs, Inc.) by extending the time delay up to $\sim 4 \mathrm{~ns}$ between pump and probe pulses that interact with the sample. ${ }^{3}$ The white-light probe was generated via the same method as described above; however, the pump and probe pulses were not further temporally compressed. The cross-correlation time for the extended time window setup was measured to be $\sim 600 \mathrm{fs}$ in 1-mm-pathlength sample solution. A spectrograph (Acton SpectraPro SP-2356, Princeton Instruments, Inc.) dispersed the transmitted probe with a 300 grooves $/ \mathrm{mm}, 500 \mathrm{~nm}$ blaze wavelength reflective grating before imaging on a front-illuminated Lumogen UV-coated CCD array camera (PIXIS:100F, Princeton Instruments).

Quantum calculations. Gaussian 09 software $^{14}$ was used to perform quantum calculations on dimethylxylindein. The B3LYP functional and $6-311+\mathrm{G}(\mathrm{d}, \mathrm{p})$ basis sets were used to with densityfunctional theory (DFT) and time-dependent (TD)-DFT methods to perform ground and excited state calculations, respectively. The unrelaxed energy values were obtained by performing a 
ground state $\left(S_{0}\right)$ geometrical optimization via DFT, followed by TD-DFT energy calculation of the first 10 singlet and first 10 triplet states at that optimized geometry. The relaxed energy values for $S_{1}, T_{2}, T_{1}$ states (in parentheses, Figure 4) were taken by performing a TD-DFT geometrical optimization relative to the electronic singlet excited state with energy calculations of the first 10 singlet and first 10 triplet states, ${ }^{15}$ also helping to evaluate frontier molecular orbitals (FMOs) of the relaxed excited state(s). To implicitly model DCM solvent, the default solvent parameters were employed with the polarizable continuum model using integral equation formalism variant (IEFPCM). Theoretical Raman spectrum from DFT calculations (Figure S9) with representative vibrational motions (see Figure S10 and Table S1 below) provide useful comparisons with experimental FSRS spectra. To evaluate the FMOs upon photoexcitation, the TD-DFT energy calculation was performed on the ground-state DFT-optimized structure. ${ }^{12}$

Notably, for the planar structure of dimethylxylindein in DCM, the calculated $S_{1} / T_{2}$ states have overall $\pi \pi^{*}$ character with weak $n \pi^{*}$ character on the methoxy and carbonyl groups closest to the tail. From the FMO analysis, $S_{2-4}$ states with increasing $n \pi^{*}$ characters could contribute to extra ISC pathways (e.g., to the energetically close $T_{5}$ with $\pi \pi^{*}$ character) from higher-lying electronic states (e.g., $400 \mathrm{~nm}$ excitation case) due to more effective orbital mixing. ${ }^{16,17}$ To evaluate the coordinate-dependent energy levels, a dihedral angle around the central core of dimethylxylindein (see Figure S11 inset) was continuously twisted and frozen to perform the DFT geometrical optimization, followed by TD-DFT energy calculations at each dihedral angle to represent the chromophore structures with an increasing degree of nonplanarity. ${ }^{6}$ Similar to our previous calculations on xylindein, the dimethylxylindein plot shows that the energy difference between the planar structure $S_{1} / T_{2}$ states is $\sim 0.18 \mathrm{eV}$, which shrinks to $\sim 0.073 \mathrm{eV}$ at $100^{\circ}$ (see relevant energy levels in Figure 4, main text). 
Upon further relaxation in the excited state that could be modeled by high-level calculations of the nonplanar chromophore conformations, the energy difference between $S_{1}$ and $T_{2}$ states could be further reduced to facilitate the major ISC pathway depicted in Figure 4. For corroboration, we calculated the energy difference between $S_{1}$ ' and $T_{2}$ ' states to be $0.05 \mathrm{eV}$ for the planar structure, while the corresponding $S_{1}{ }^{\prime} / T_{2}$ ' (relaxed) energy gap also shrinks relative to $S_{1} / T_{2}$ (unrelaxed) for xylindein. ${ }^{6}$ In essence, both the excited-state energy relaxation and ultrafast chromophore conformational motions that deviate from ring-coplanarity could reduce the energy difference between adjacent singlet and triplet states, highlighted by the above-mentioned systematic comparison between unrelaxed and relaxed $S_{1}$ and $T_{2}$ states that constitute the main ISC pathway for the photoexcited dimethylxylindein in DCM (see Figure 4 and main text). Such a mechanism likely applies to the higher-lying singlet and triplet states $\left(S_{\mathrm{n}}\right.$ and $\left.T_{\mathrm{n}+1}\right)$ as well via spin-orbit coupling, which underlies the excitation wavelength dependence of triplet state formation on ultrafast timescales (more triplet state with bluer excitation, see Figures 1a and S3a). 


\section{$\underline{\text { SI Figures }}$}
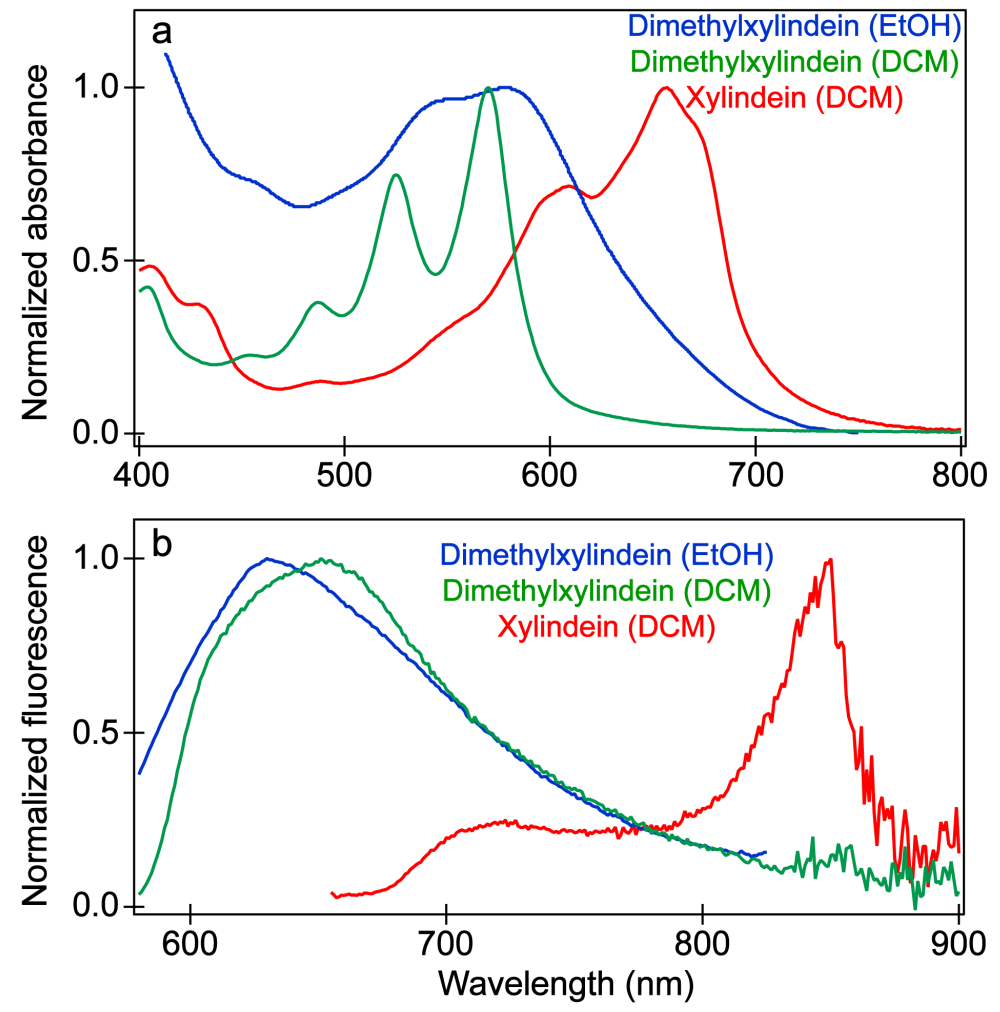

Figure S1. Normalized steady-state (a) absorption and (b) emission spectra of dimethylxylindein in DCM (green) and ethanol (blue), and xylindein in DCM (red). The excitation wavelengths for the emission spectra of dimethylxylindein and xylindein were 560 and $640 \mathrm{~nm}$, respectively.

The broad absorption profiles of dimethylxylindein and xylindein spanning most of the visible spectrum are beneficial for optoelectronic and light-harvesting applications. In both spectra, a pronounced vibronic progression is observed to the blue side with the vibronic peaks of dimethylxylindein well-resolved (see sharp peaks in green trace, Figure S1a). For xylindein, these vibronic peaks were previously fit with two tautomers (i.e., hydrogen away-from-tail and hydrogen toward-tail) representing whether the hydroxy groups are pointed toward or away from the central carbonyl groups (i.e., the two carbonyls farthest from the extended tails, see Scheme 1). ${ }^{2,6}$ The 
dimethylxylindein peak maximum $(568 \mathrm{~nm})$ is blue-shifted from xylindein $(656 \mathrm{~nm})$ by $\sim 100 \mathrm{~nm}$ due to a weakened hydrogen $(\mathrm{H})$-bond network upon methyl substitution. The H-bond network has been implicated to play a key role in the (photo)stability of these pigments when exposed to air, both dimethylxylindein and the deprotonated xylindein show drastically reduced stability with a significant absorption loss over time. ${ }^{3}$ The triplet state formation of dimethylxylindein in DCM also affects the apparent chromophore stability by generating reactive oxygen species (main text).

The fluorescence quantum yields of dimethylxylindein and xylindein are both low at $0.7 \%$ and $<0.1 \%$, respectively, in DCM. ${ }^{3,4}$ The broad fluorescence profile of dimethylxylindein displays a small Stokes shift (Figure S1, green traces) assigned to the relaxed singlet excited state, while its unusual broadness is in accord with intrinsic heterogeneity of the chromophore (e.g., observed beneath the absorption profile in Figure S1a). ${ }^{12,18}$ While not expected to be pronounced at room temperature, the phosphorescent population due to intersystem crossing (ISC) may contribute to the emission spectrum (e.g., $T_{1} \rightarrow S_{0}$ plus $S_{1} \rightarrow S_{0}$ ). ${ }^{19}$ The fluorescent state is termed a charge-transfer (CT) state according to the detailed fs-TA, GS- and ES-FSRS measurements on dimethylxylindein, as well as the composition of FMOs from quantum calculations. ${ }^{14}$

Interestingly, xylindein shows two fluorescence peaks at 709 and $846 \mathrm{~nm}$ assigned to the protonated state $\left(\mathrm{PA}^{*}\right)$ and excited-state intramolecular proton transfer (ESIPT) product, wherein the dissociable proton of the hydroxy group jumps to the nearby carbonyl group on ultrafast timescales. ${ }^{6}$ The sensitivity of the fluorescence measurement drops around $850 \mathrm{~nm}$, causing the steep cutoff in the xylindein spectrum (red trace, Figure S1b). An excitation-dependent change of the fluorescence spectrum reveals a key finding: bluer-wavelength light excites both the H-awayfrom-tail and H-toward-tail tautomers with nearly equal fluorescence peak intensities, whereas redder-wavelength light mostly excites the H-away-from-tail tautomer (supporting ESIPT from 
the hydroxy groups to the central carbonyl groups) and lead to a much stronger $846 \mathrm{~nm}$ peak. ${ }^{6}$ This sharp contrast between xylindein and dimethylxylindein substantiates the major effect of methylation $^{20}$ at two key atomic sites of the fungal pigment on the emission properties and more fundamentally, the excited-state electronic and structural dynamics of these closely related chromophores in condensed phase. ${ }^{12}$

A series of concentration-dependent steady-state absorption measurements were taken for dimethylxylindein in both $\mathrm{DCM}$ (from $\mathrm{OD}=0.005 / \mathrm{mm}$ up to $1.2 / \mathrm{mm}$ ) and ethanol (from $\mathrm{OD}=0.01 / \mathrm{mm}$ up to $0.2 / \mathrm{mm}$ due to poor solubility), which show a slightly reduced main absorption bandwidth in the visible region, instead of a growing red-shifted absorption peak due to aggregate formation. ${ }^{4,6}$ With photoexcitation on the bluer side of the main absorption peak (e.g., see Figure 1), our fs-TA spectra thus mainly track the monomeric dimethylxylindein species in solution following photoexcitation. Moreover, the dimethylxylindein spectra in solution differ from its thinfilm spectrum ${ }^{3}$ that shows a long tail of the electronic absorption band from $\sim 635$ to $800 \mathrm{~nm}$, which is clearly red-shifted from the dimethylxylindein in ethanol case (Figure S1a, blue trace). The associated thin-film photoluminescence peak at $\sim 710 \mathrm{~nm}$ also represents a notable redshift from its emission peak in ethanol ( $\sim 630 \mathrm{~nm}$, Figure S1b, blue trace). Again, this result indicates that in difference from xylindein that tends to aggregate at high concentration $(\mathrm{OD}>1 / \mathrm{mm})$ in solution and exhibits a similar red-shifted absorption peak that resembles xylindein thin films, ${ }^{4,6}$ dimethylxylindein with two peripheral methoxy groups (see Scheme 1) does not display notable aggregation behavior in solution (Figures 1a, S3, and S5). 

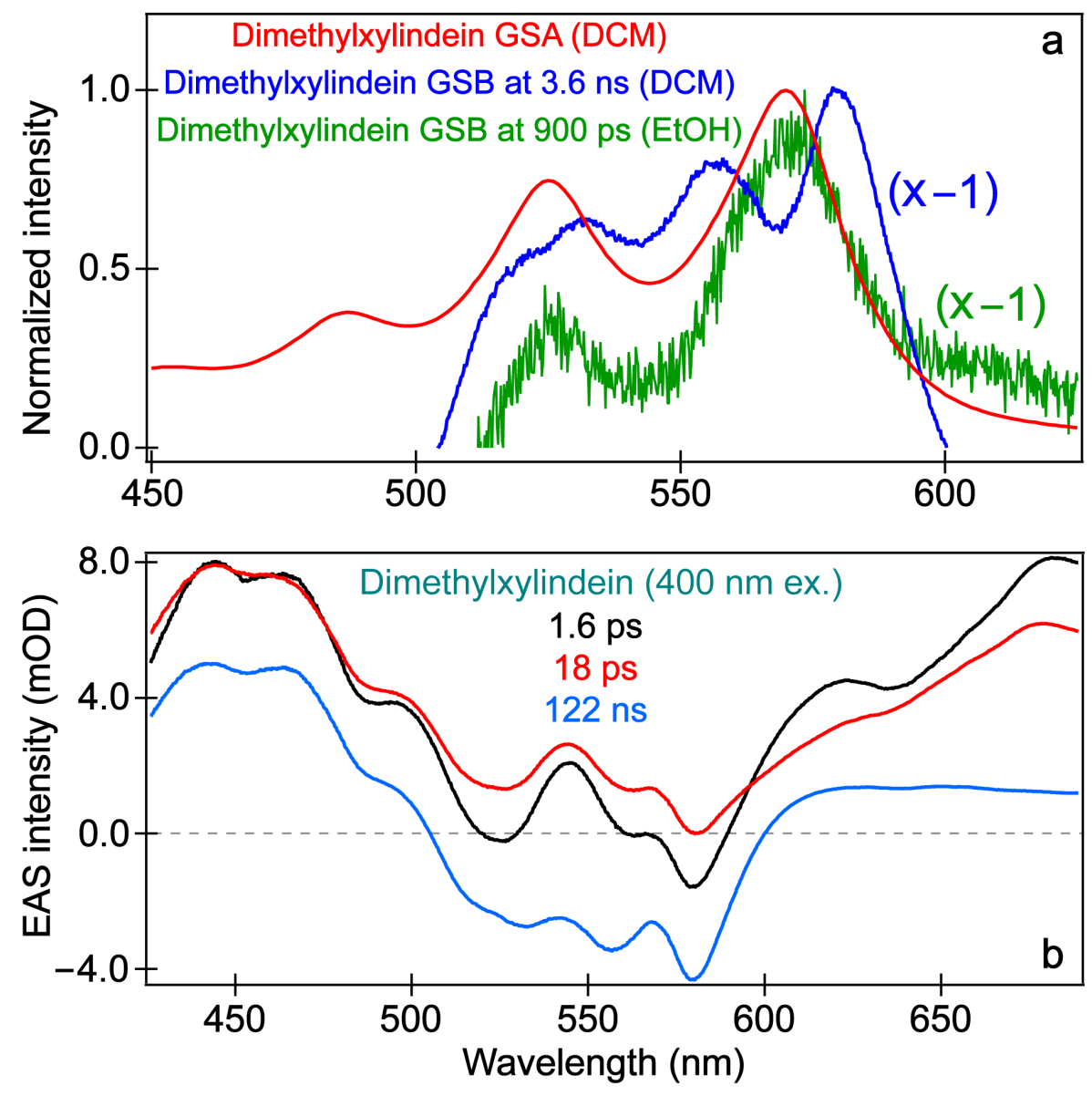

Figure S2. (a) Comparison of the normalized dimethylxylindein spectral profiles between the ground-state absorption (GSA) in DCM (red), ground-state bleaching (GSB) in DCM (blue), and GSB in ethanol (green). Following $400 \mathrm{~nm}$ excitation, the GSB traces were taken from fs-TA experiments at $3.6 \mathrm{~ns}$ in DCM and $900 \mathrm{ps}$ in EtOH, respectively. Since the GSB signal intensities from fs-TA spectra are negative, they are inverted $(\times-1)$ and displayed as positive signal to compare with the steady-state absorption band and peak positions. (b) Evolution-associated spectra (EAS) from global analysis of dimethylxylindein in DCM following $400 \mathrm{~nm}$ excitation. The lifetimes for the sequential kinetic components (black $\rightarrow$ red $\rightarrow$ cyan) are listed in the inset.

Notably, the GSB of dimethylxylindein in DCM (Figure S2a, blue trace) is red-shifted from the GSA peak location (Figure S2a, red trace) by $\sim 10 \mathrm{~nm}$ while maintaining a similar spectral 
profile. In addition, the vibronic coupling pattern is altered as the energy difference between the main peak and first blue shoulder is $\sim 700 \mathrm{~cm}^{-1}$ for GSB in DCM, while this energy difference is $\sim 1504 \mathrm{~cm}^{-1}$ for GSA in DCM. This observation refutes an argument that the observed GSB band is simply due to the decay of an overlapping blue ESA band, because if the significantly reduced vibronic peak spacing arose from a much stronger overlap between the positive ESA and negative GSB around $\sim 530 \mathrm{~nm}$ than that around $570 \mathrm{~nm}$, the apparent peak intensity of the first blue shoulder would decrease to a large extent, which does not agree with the experimental spectrum (blue trace above, the first blue shoulder peak exhibits a relative intensity increase with respect to the main peak). Since the GSB of dimethylxylindein in DCM was taken at a long time delay (3.6 ns, see Experimental methods above for details), it likely represents a chromophore subpopulation with a proclivity to undergo ISC and prolong the excited state decay, in accord with long lifetimes of triplet states (main text). ${ }^{3,21}$

Typically, the GSB band recovers as the excited-state population returns to the ground state, which is the case for xylindein in DCM (Figures $1 b$ and $2 b, f) .{ }^{6}$ In ethanol, the dimethylxylindein GSB band does not fully recover (Figure S5b, blue trace) within the detection time window of $\sim 900$ ps (Figure S5a), though the 2.1 ns lifetime is characteristic of a fluorescent state (e.g., radiative $S_{1} \rightarrow S_{0}$ transition). ${ }^{12}$ Furthermore, the GSB band of dimethylxylindein in EtOH (Figure S2a, green trace) is not red-shifted and largely matches the GSA band (Figure S2a, red trace), revealing that most of the chromophore population does not undergo ISC to triplet state in the more polar solvent. ${ }^{22,23}$ In other words, H-bonding interactions between $\mathrm{EtOH}$ and dimethylxylindein (particular the $\mathrm{C}=\mathrm{O}$ groups) help to maintain a more planar chromophore structure without discernible subpopulations. This systematic analysis exposes the "hidden" intrinsic ground-state heterogeneity of dimethylxylindein population in DCM that is more likely 
to undergo ISC in the excited state (see Figure 4 in main text for an overall energy level diagram). With steady-state absorption spectroscopy alone, this distinct subpopulation cannot be observed as it is embedded within the broad absorption profile. Nonplanar molecular structures have been implicated to promote ISC in many systems, ${ }^{24}$ so this dimethylxylindein subpopulation likely consists of a distorted conformation to some extent in the electronic ground state (at thermal equilibrium). This point is also in accord with the DFT-based quantum calculation results and the central-dihedral-angle-dependent energy change trend in the excited state (e.g., a nonplanar chromophore leads to a red-shifted absorption peak from the planar case, see Figure S11 below for representative calculations of pertinent energy levels including the ground state $S_{0}$ ).

In particular, the EAS (see Figure S2b above) instead of DAS (Figure 2a in main text) from global analysis of dimethylxylindein in DCM following $400 \mathrm{~nm}$ excitation confirms the correlated red and blue ESA bands, and the significant spectral overlap between a broad positive ESA band and a structured negative GSB band in the visible region. Using the sequential (Figure S2b) and parallel (Figure 2a) kinetic models in EAS and DAS, respectively, we highlight the faster decay of red ESA band above $\sim 600 \mathrm{~nm}$ than blue ESA band below $\sim 500 \mathrm{~nm}$. 

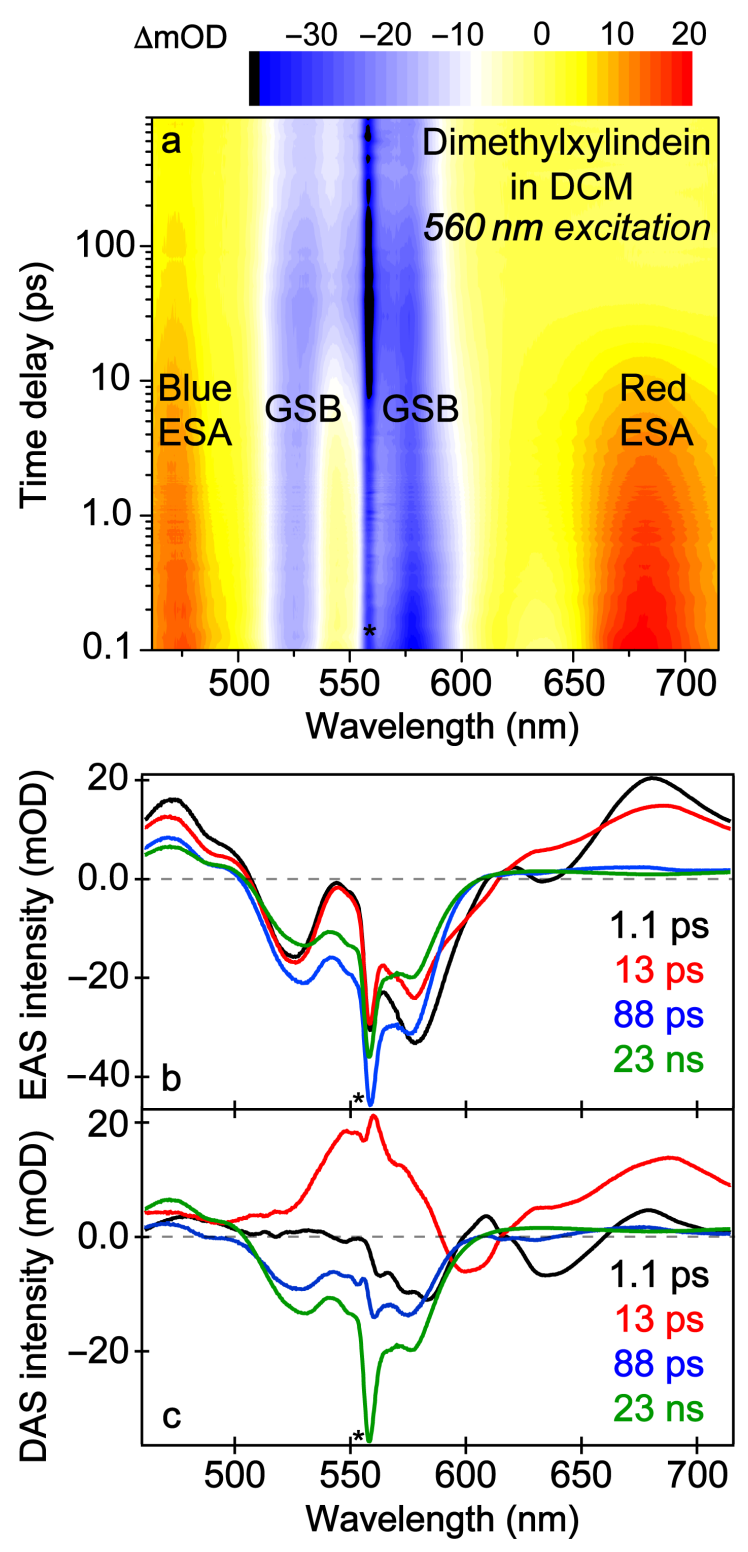

Figure S3. Transient electronic features of dimethylxylindein in solution. (a) Semilogarithmic contour plot of the fs-TA spectra of dimethylxylindein in DCM after $560 \mathrm{~nm}$ excitation (up to $\sim 900$ ps time delay). Prominent features are labeled with intensity colorbars shown above. Global analysis results are represented by (b) evolution-associated spectra (EAS) and (c) decay-associated spectra (DAS). A four-component model (black $\rightarrow$ red $\rightarrow$ blue $\rightarrow$ green) accurately fits fs-TA data with the color-coded lifetimes listed. A sharp negative signal at $\sim 560 \mathrm{~nm}$ is due to scattering of the actinic pump (marked by black asterisks). The zero change of OD is shown as dashed gray line. 
The fs-TA spectra of dimethylxylindein in DCM with $560 \mathrm{~nm}$ excitation was performed as a control experiment to evaluate the effect of excitation wavelength on the ensuing excited-state energy dissipation. A redder excitation wavelength was selected to be more resonant with the $\mathrm{S}_{0} \rightarrow \mathrm{S}_{1}$ transition of the chromophore, though the detection time window was limited to $900 \mathrm{ps}$ (see Experimental methods for details for the pertinent TA setup). The 2D contour plot shows several similarities to the TA spectra with $400 \mathrm{~nm}$ excitation (Figure 1a, main text). Interestingly, the GSB can be observed from time zero of $560 \mathrm{~nm}$ excitation, contrasting the $400 \mathrm{~nm}$ case where the negative GSB is spectrally overlapped with positive ESA bands at early times. Furthermore, the GSB is still red-shifted from the GSA (like Figure S2a) and does not recover within $\sim 1$ ns.

For dimethylxylindein in DCM, red ESA band appears at 683 and $679 \mathrm{~nm}$ after 560 and 400 $\mathrm{nm}$ excitation, respectively. Since we mainly attributed this red ESA to an $S_{1} \rightarrow S_{\mathrm{n}}$ transition, the noticeable blueshift of red ESA peak wavelength with a bluer excitation (e.g., $400 \mathrm{~nm}$ ) implies that an ultrafast internal conversion (within our cross-correlation time of $\sim 140 \mathrm{fs}$ ) reaches a lowerlying $S_{1}$ ' state than the redder-excitation-accessed $S_{1}$ state. ${ }^{25}$ It is thus reasonable to observe the different initial decay component of red ESA, 1.7 ps (14\% amplitude weight, Figure 2c) after 400 $\mathrm{nm}$ excitation and $500 \mathrm{fs}$ ( $21 \%$ amplitude weight, Figure S4a) after $560 \mathrm{~nm}$ excitation, indicating that $S_{1}$ ' takes longer with a reduced weight to undergo the spin-conserved relaxation (Figure 4). Similarly, the initial blue ESA peak wavelength also exhibits a notable blueshift from $473 \mathrm{~nm}$ (after $560 \mathrm{~nm}$ excitation) to 445, $460 \mathrm{~nm}$ (after $400 \mathrm{~nm}$ excitation), which agrees with a slightly different excited state accessed by direct pump $\left(S_{0} \rightarrow S_{1}\right)$ or an indirect route $\left(S_{0} \rightarrow S_{\mathrm{n}} \rightarrow S_{1}{ }^{\prime}\right)$. The shift could also be affected by accessing different triplet states upon bluer and redder excitations on ultrafast timescales, which is supported by the relaxed and unrelaxed FMOs from quantum calculations (see above). 
A four-component model was required for the global analysis of TA spectra, ${ }^{26,27}$ including one additional component ( $88 \mathrm{ps}$ ) versus global analysis results with $400 \mathrm{~nm}$ excitation. The three conserved lifetimes ( $\sim 1.1 \mathrm{ps}, 13 \mathrm{ps}$, and $23 \mathrm{~ns}$ in Figure S3b,c) are similar to those retrieved with $400 \mathrm{~nm}$ excitation ( $\sim 1.6 \mathrm{ps}, 18 \mathrm{ps}$, and $122 \mathrm{~ns}$ in Figures $2 \mathrm{a}$ and $\mathrm{S} 2 \mathrm{~b})$, confirming the significant overlap between ESA and GSB bands in the visible spectral region. Using the fluorescence quantum yield of dimethylxylindein in $\operatorname{DCM}\left(\Phi_{r} \approx 0.7 \%\right.$, Eq. 1$)$ and a typical fluorescence lifetime of 2-5 ns $\left(k_{r}^{-1}\right)$, the apparent $S_{1}$ lifetime $\left(k_{F}^{-1}\right)$ can be estimated to be $14-35$ ps (Eq. 3), closely matching our experimental observation of the fluorescent state being dominated by nonradiative relaxation $\left(k_{r} \ll k_{n r}\right)^{28,29}$

$$
\begin{aligned}
& \Phi_{r}=\frac{k_{r}}{k_{r}+k_{n r}} \\
& k_{F}=k_{r}+k_{n r} \\
& k_{F}^{-1}=\frac{\Phi_{r}}{k_{r}}
\end{aligned}
$$

The final component from TA spectra global analysis is reduced by $\sim 100 \mathrm{~ns}$, likely influenced by a combination of two factors. First, the shortened experimental time window of 900 ps limits the evaluation of these long-lived decay occurring beyond the time window. Second, the triplet yield with $560 \mathrm{~nm}$ excitation is reduced due to a diminishment of ISC pathways that originate from higher-lying electronic states following $400 \mathrm{~nm}$ excitation (e.g., $S_{\mathrm{n}} \rightarrow T_{\mathrm{x}}$ ). As this final component represents an average lifetime of $S_{1} / T_{1}$ states, a lifetime reduction suggests more of a fluorescent population with a typical lifetime on the ns timescale ${ }^{12}$ or, vice versa, less of a phosphorescent population with typical lifetimes extending into the micro-to-millisecond regime. ${ }^{11}$ Another observable to support this interpretation is displayed by blue ESA band intensity, characterizing more of the triplet state(s), and the red ESA intensity, characterizing mostly the singlet state(s). From the time zero of photoexcitation, blue ESA is less intense than red ESA after $560 \mathrm{~nm}$ 
excitation (Figure S4a) while these ESA bands show nearly equal intensities after $400 \mathrm{~nm}$ excitation (Figure 2c), which indicates less triplet formation with redder excitations.

Target analysis using a hybrid parallel and sequential kinetic model was performed resulting in the species-associated spectra (SAS) ${ }^{26,30}$ Specifically, the model consists of three states: (1) an initially populated singlet excited state $\left(S_{1}\right),(2)$ a stabilized charge-transfer state $\left(S_{1}{ }^{\prime}\right.$ '), and (3) a triplet state $\left(T_{1}\right)$. The target analysis model is similar to the proposed model in Figure 4, wherein $S_{1}$ can bifurcate into $S_{1}$ " or $T_{2}$ (then rapidly relaxing into a lower-lying state $T_{1}$ ). Meanwhile, the $S_{1}$ " population mainly undergoes nonradiative relaxation to $S_{0}$. A variety of branching ratios were stipulated to evaluate the hybrid model, and a majority of these diverse branching ratios reached convergence which supports the robustness of our proposed model in Figure 4 (see main text). 


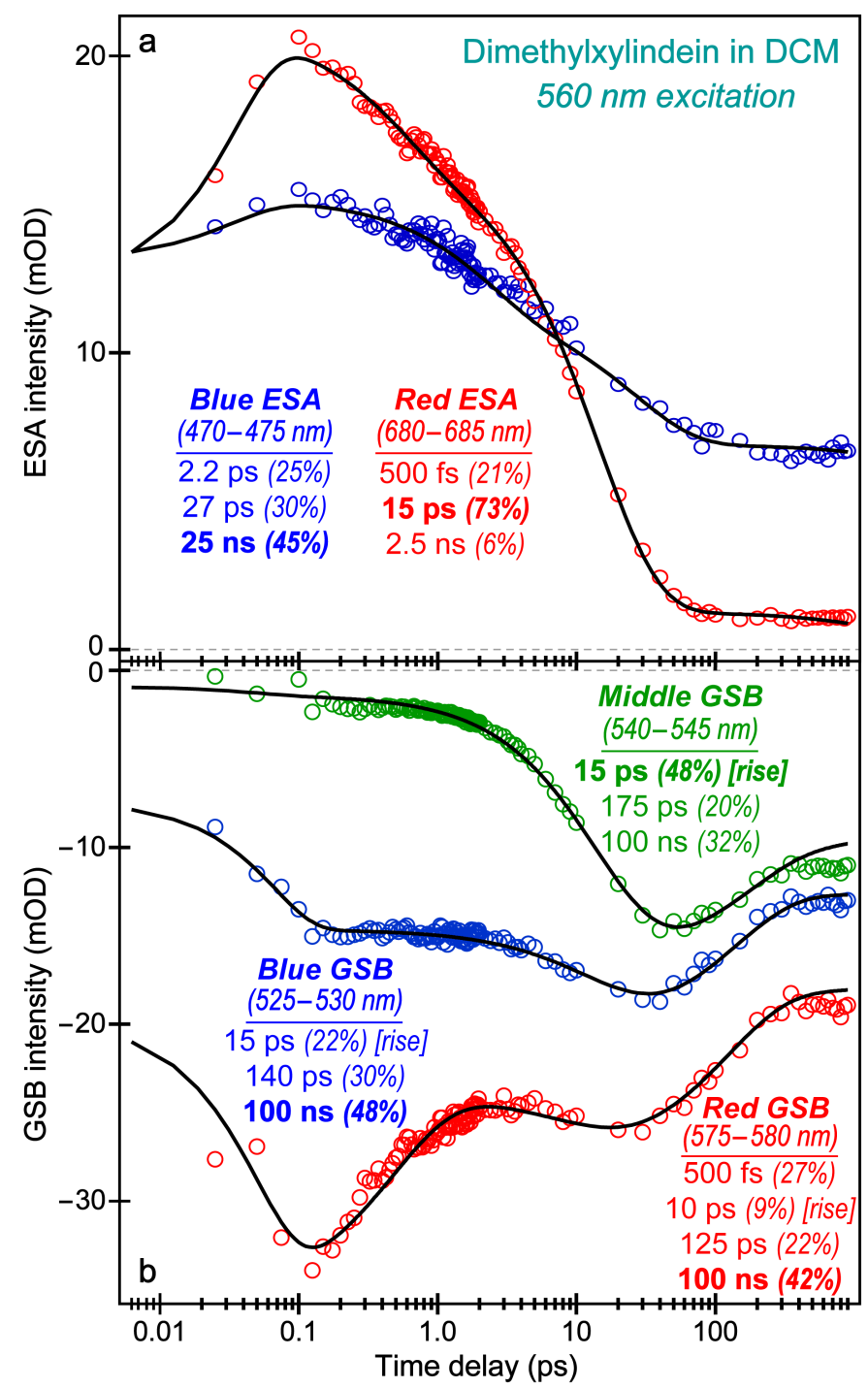

Figure S4. The average integrated intensity of (a) ESA and (b) GSB features from fs-TA spectra of dimethylxylindein in DCM up to $\sim 900 \mathrm{ps}$ after $560 \mathrm{~nm}$ excitation. The multiexponential leastsquares fits are shown as black solid curves overlaid with data points (open circles). The colorcoded time constants and probe spectral integration regions are listed in the inset, and the major component is bolded for each TA feature. The zero change of OD is shown as a dashed gray line.

The probe-dependent intensity dynamics of dimethylxylindein in DCM with $560 \mathrm{~nm}$ excitation are reminiscent of the $400 \mathrm{~nm}$ excitation case (Figure 2c,e in main text). With the redder excitation, following the cross-correlation time of $\sim 140$ fs in TA setup with a 900-ps detection time window, ${ }^{6}$ 
the first component assigned to CT state stabilization occurs faster and with a larger amplitude weight for both blue and red ESA, 2.2 ps (25\%) and 500 fs (21\%) in Figure S4a, than those after bluer excitation, $3.0 \mathrm{ps}(15 \%)$ and $1.7 \mathrm{ps}(14 \%)$ in Figure 2c. The dynamics reveal that direct pump to $S_{1}$ state makes CT relaxation more efficient, while the amplitude weights and lifetimes of blue/red ESA features primarily tracking the triplet/singlet states on the ns timescale are reduced. Besides reduced ISC pathways that limit triplet formation, efficient CT relaxation may further quench ISC and could be examined by more experiments with additional excitation wavelengths. For example, after $580 \mathrm{~nm}$ excitation (redder than $560 \mathrm{~nm}$ ) which may better excite any potential aggregated species of dimethylxylindein in DCM (if some aggregation could occur at this low solute concentration), we observed a less intense blue ESA band with a 9 ns decay time constant that is further decreased from the corresponding $25 \mathrm{~ns}$ component in Figure S4a. Notably, the latter component is already smaller than its $40 \mathrm{~ns}$ counterpart after $400 \mathrm{~nm}$ excitation in Figure $2 \mathrm{c}$ (main text), which exhibits the most triplet state formation on ultrafast timescales. These results indicate that no significant aggregated species or contaminants ${ }^{2,4}$ contribute to our spectral observation of monomeric dimethylxylindein in solution with largely conserved TA features (Figures 1a, S3, S5).

With less spectral overlap in the TA contour plot, the GSB feature is observed from time zero, yet it still displays an unusual rise component (prior to $\sim 50 \mathrm{ps)}$ ) with a conserved time constant (Figure S4b). The apparent rise of the dimethylxylindein GSB intensity with a $10-15$ ps time constant provides convincing evidence that ultrafast structural distortions lead to a splitting of the excited-state population with distinct and overlapping negative GSB and positive ESA bands, ${ }^{12,31}$ especially when the GSB can be tracked reliably throughout fs-TA experiments (Figures 1, 2, and S2-S4). Our spectral simulations with decaying ESA and GSB bands showed that the only way to reproduce the distinct rise pattern in Figures $2 \mathrm{e}$ and $\mathrm{S} 4 \mathrm{~b}$ is to have the ESA decay with a conserved 
10-20 ps time constant but a larger weighted amplitude than the concomitant GSB decay, while the overall amplitude magnitude of the GSB band with the 40-100 ns decay time constant still surpasses the overlapping ESA band (otherwise the TA signal would be positive instead of negative as observed experimentally in the visible spectral region).

A $125-175$ ps decay component (20-30\% amplitude weight) of GSB band is consistently observed within a broad spectral region (Figures S3a and S4b) following $560 \mathrm{~nm}$ excitation, representing a longer process of nonradiative return to the ground state, likely due to molecular rotational motions. ${ }^{20,32}$ These values fall within the estimated range using Debye theory to derive molecular rotational time constant. ${ }^{33,34}$ Using short axis (from methoxy to methoxy, $r \approx 6.1 \AA$ ) and long axis (from methyl groups on both tail ends, $r \approx 9.7 \AA$ ) of the dimethylxylindein structure (see Scheme 1 in main text), the calculated time constants are $\sim 95$ and 385 ps, respectively, in DCM (viscosity is 0.43 centipoise at $20{ }^{\circ} \mathrm{C}$ ) ${ }^{20}$ Notably, the $120 \mathrm{ps}$ component shows up as a minor rise after $400 \mathrm{~nm}$ excitation (Figure 2e) due to a larger intensity magnitude of ESA than GSB, whereas an inversed relative intensity leads to the usual $\sim 120$ ps decay as the excited-state population relaxes back to the ground state in a spin-conserved manner (Figure S4). These dynamic spectral components may be influenced by the nearby scattering from actinic pump $(560 \mathrm{~nm})$, likely explaining the invariant last time constant of $100 \mathrm{~ns}$ across the GSB region (Figures S3a and S4b). We further removed the actinic pump scattering from TA spectra before global analysis, ${ }^{35}$ and obtained similar EAS and DAS plots as Figure S3b,c, except a sharp dip at $\sim 560 \mathrm{~nm}$. The retrieved lifetimes are $1.1 \mathrm{ps}, 14 \mathrm{ps}, 88 \mathrm{ps}$, and $32 \mathrm{~ns}$, which closely match the aforementioned lifetimes of $1.1 \mathrm{ps}, 13 \mathrm{ps}, 88 \mathrm{ps}$, and $23 \mathrm{~ns}$. Above all, the conserved GSB dynamic pattern particularly after $\sim 2$ ps and correlation with the blue and red ESA bands (Figure S4) corroborate the aforementioned assignment of excited-state relaxation processes for dimethylxylindein in DCM. ${ }^{12}$ 

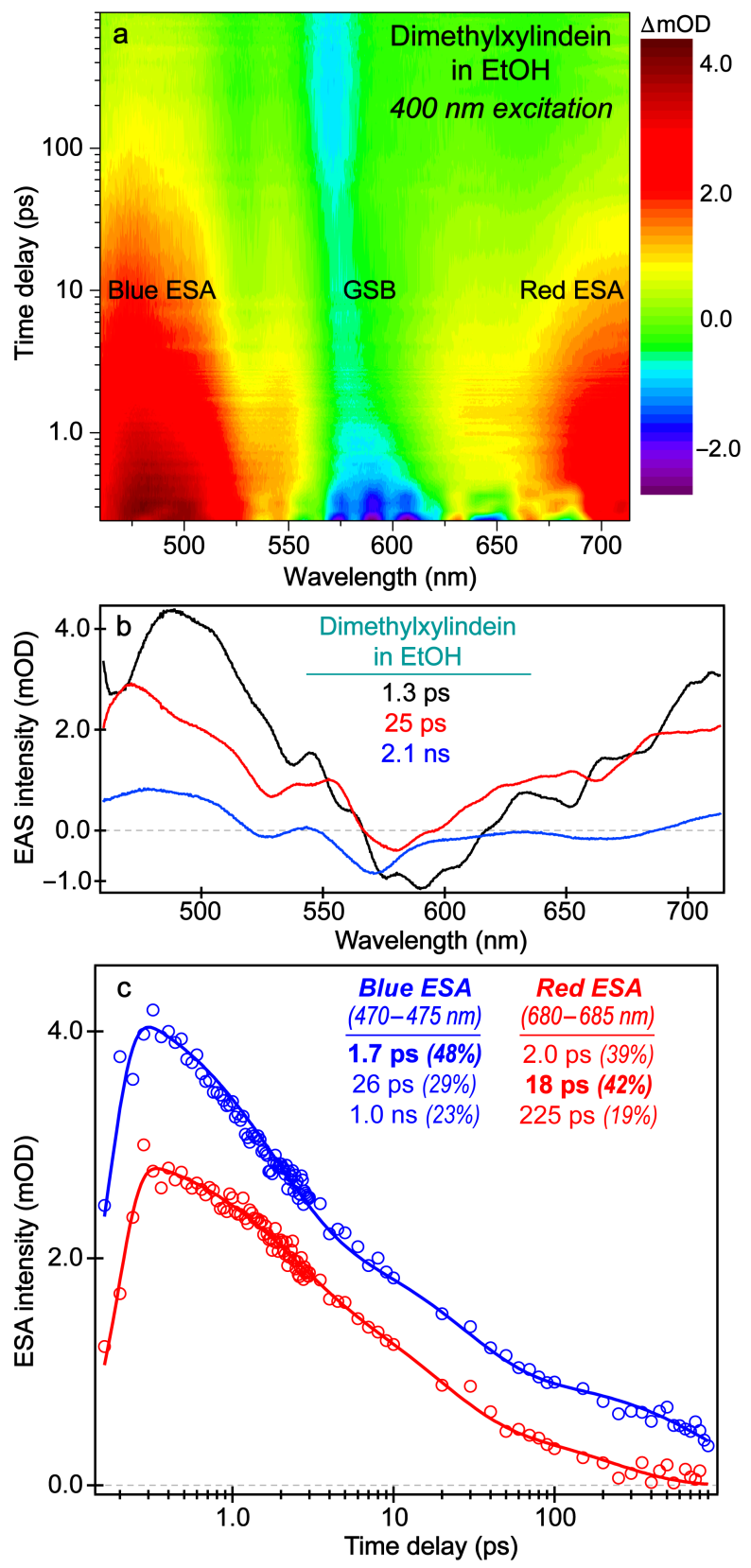

Figure S5. Time-resolved electronic spectra of dimethylxylindein in ethanol (EtOH). (a) Semilogarithmic contour plot of the fs-TA spectra of dimethylxylindein in EtOH up to $\sim 900$ ps after $400 \mathrm{~nm}$ excitation. Major bands are labeled. The intensity colorbars are shown in mOD units on the right side. (b) Global analysis of the dimethylxylindein fs-TA spectra as represented by the three-component EAS (black $\rightarrow$ red $\rightarrow$ blue) with the color-coded lifetimes listed in the inset. (c) Blue and red ESA peak intensity dynamics of dimethylxylindein in ethanol. The multiexponential 
least-squares fits (color-coded solid curves) are overlaid with data points (open circles). The associated lifetimes and probe spectral integration regions are listed in the inset with major component bolded for each ESA feature. The zero change of OD is shown as the dashed gray line.

Notably, no triplet state formation was detected for dimethylxylindein in ethanol (see Figure S2 and the ensuing discussions above) while the sample solution remains transparent (no floating impurities). ${ }^{2}$ We propose that intricate solute-solvent interactions involving polar solvents could raise the energy of the triplet states above the respective singlet states, thus making the ISC improbable. Several other supporting interpretations for the lack of triplet formation could be derived from fs-TA data. Similar to the aforementioned spectral comparison between the 400 and $560 \mathrm{~nm}$ excitation cases for dimethylxylindein in DCM, ultrafast relaxation within the CT state is enhanced in the more polar solvent ethanol (than DCM) $)^{23,36}$ which could effectively compete with the ISC process. Our previous investigation on the structural dynamics of a methylated derivative of a popular photoacid pyranine, 8-methoxypyrene-1,3,6-trisulfonate (MPTS), found that the formation of a CT state becomes more prominent as the solvent polarity increases. ${ }^{20}$ The CT state stabilization may quench ISC to a certain degree, though a more extensive study of dimethylxylindein in a variety of solvents is required for further insights. Though the lifetime of the intermediate decay component (18-26 ps, Figure S5c) is slightly longer in ethanol, the amplitude weight (42-29\%) is reduced relative to that in DCM (Figure 2c), which may be owing to the enhanced CT state stabilization that diminishes these nonplanar ring conformational motions. ${ }^{36}$ Moreover, the increased solute-solvent H-bonding interactions may further constrain the nonplanar conformational motions of dimethylxylindein in ethanol and dissipate the energy more efficiently from the lower-lying $S_{1}$ " state (e.g., nonradiative relaxation back to $S_{0}$ on the $\sim 225$ ps timescale, red trace in Figure S5c), ${ }^{6,20,37}$ which results in a lower probability for ISC. 

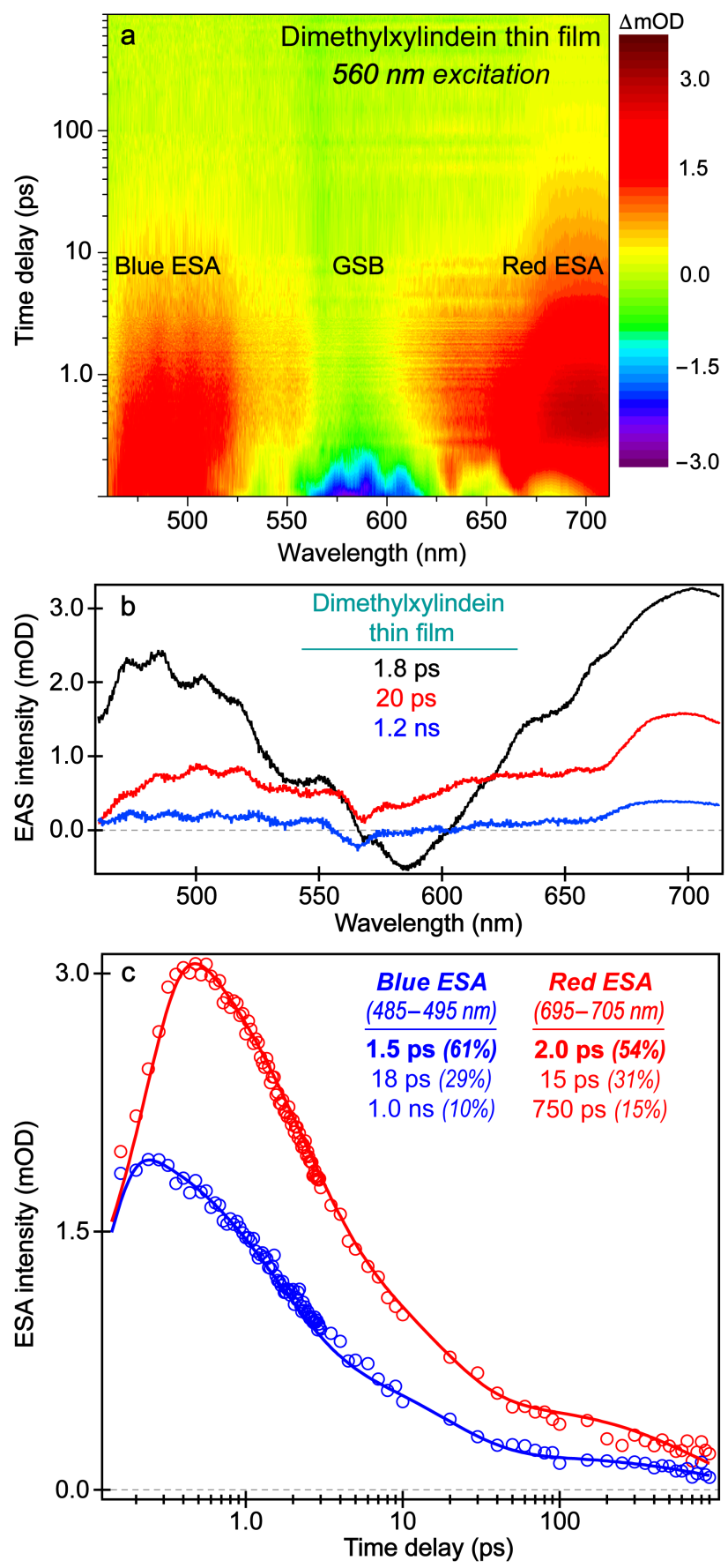

Figure S6. Time-resolved electronic spectra of dimethylxylindein thin film. (a) Semilogarithmic contour plot of fs-TA spectra of the spin-coated dimethylxylindein thin film up to $\sim 900$ ps after $560 \mathrm{~nm}$ excitation. Major electronic bands are labeled. The intensity colorbars are shown in mOD units on the right side. (b) Global analysis of the dimethylxylindein spectra as represented by the three-component EAS (black $\rightarrow$ red $\rightarrow$ blue) with the color-coded lifetimes listed in the inset. (c) 
Blue and red ESA peak intensity dynamics of thin-film dimethylxylindein. The multiexponential least-squares fits (color-coded solid curves) are overlaid with data points (open circles). The corresponding lifetimes and probe spectral integration regions are listed in the inset with the major component bolded for each ESA feature. The zero change of OD is shown as the dashed gray line.

The spin-coated dimethylxylindein thin films were probed via fs-TA measurements upon 560 $\mathrm{nm}$ excitation. Similar to the aforementioned dimethylxylindein in ethanol case (Figure S5), no significant triplet state formation was observed in thin films. This result may arise from several factors; however, the dominant factor could still be a substantial enhancement of CT state stabilization that effectively reduces the ISC probability. Moreover, it is reasonable to deduce that in the tightly "packed" solid state, nonplanar conformational motions become more restricted and thus limit spin-orbit coupling throughout the molecular framework of dimethylxylindein. ${ }^{3} \mathrm{~A}$ prominent decay on the $\sim 15 \mathrm{ps}$ timescale indicates that structural deviations from ring planarity still occur; though with no triplet state lifetime observed beyond $\sim 1 \mathrm{~ns}$, the ISC accompanying these motions is unlikely. The similarity between the thin-film and solution-phase excited state dynamics of dimethylxylindein infers that no excimers are appreciably formed in the thin-film dimethylxylindein lacking key hydroxy groups, in sharp contrast to xylindein (see Scheme 1).,

To enhance and optimize the triplet state formation in dimethylxylindein thin films, sitespecific substitutions could be suggested to promote this sustainable organic pigment as a triplet state optoelectronic material ${ }^{11}$ as follows. (1) Successive thionation of the carbonyl groups. ${ }^{38,39}$ (2) Implementation of heterocyclic nitrogens. ${ }^{40}$ (3) Decorating the peripheral moieties with halogens to promote heavy-atom effect (HAE). ${ }^{41}$ (4) A host-polymer matrix, such as polymethyl methacrylate (PMMA), may improve thin-film morphology and charge-generation efficiency., ${ }^{3,4}$ 


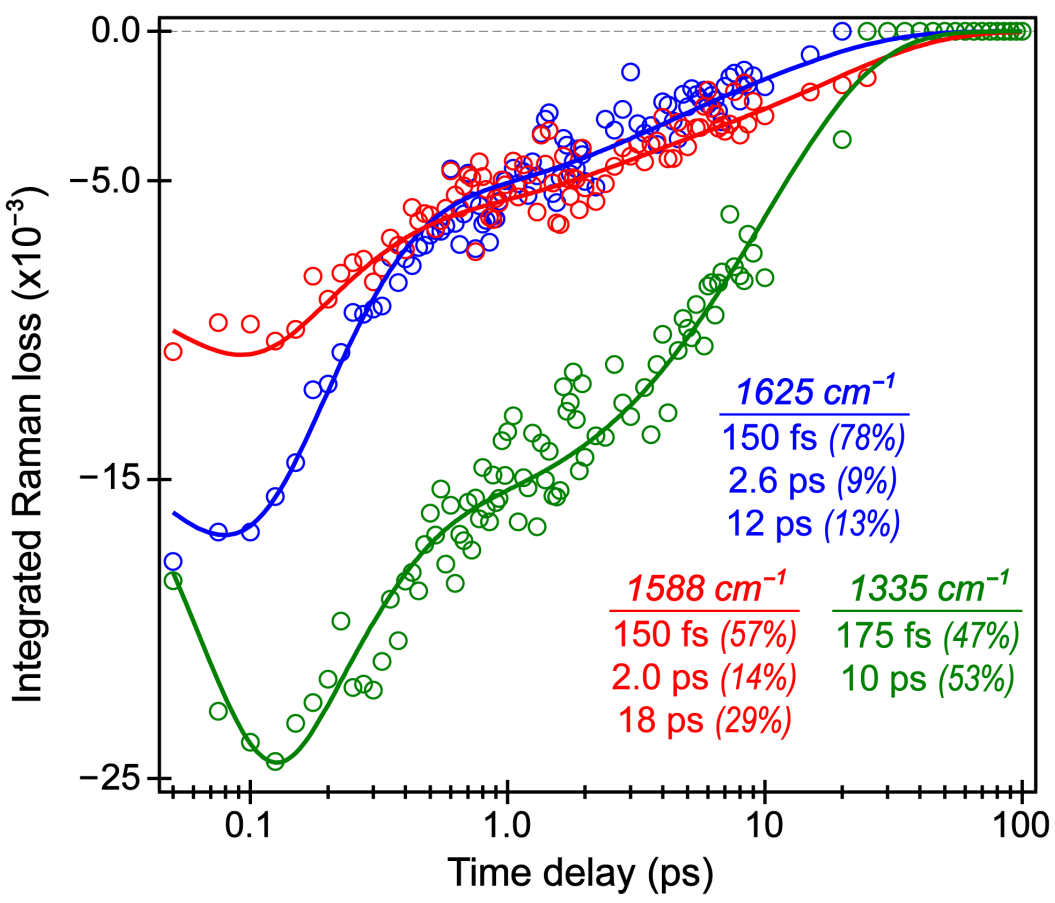

Figure S7. Peak intensity dynamics of the anti-Stokes excited-state FSRS of dimethylxylindein in DCM upon $560 \mathrm{~nm}$ excitation with a $710 \mathrm{~nm}$ Raman pump. Vibrational bands with center frequencies of $1625 \mathrm{~cm}^{-1}$ (blue), $1588 \mathrm{~cm}^{-1}$ (red), and $1335 \mathrm{~cm}^{-1}$ (green) were fit with gaussian functions. The integrated intensity data points (Raman loss with negative sign, circles) are overlaid with the least-squares exponential fits (color-coded solid curves) on a logarithmic timescale. The retrieved time constants and amplitude weight percentages are listed accordingly.

Various Raman pump conditions in FSRS were attempted to achieve the most intense excitedstate vibrational peaks. ${ }^{8,12}$ With Raman pump wavelengths tuned to 650 and $675 \mathrm{~nm}$ (see Experimental methods above), the Stokes and anti-Stokes FSRS experiments were performed with several well-resolved peaks. ${ }^{8,42}$ With a $710 \mathrm{~nm}$ Raman pump and broadband Raman probe on the anti-Stokes side, we observed the strongest excited-state Raman signal. Resonance enhancement with red ESA band yields similar vibrational dynamics on the ps timescale to those observed from fs-TA (Figure 2), with the addition of a pronounced $\sim 150$ fs time constant due to initial Franck- 
Condon relaxation that leads to a large change of electric polarizability. ${ }^{8,43}$ Several remaining prominent peaks from the ES-FSRS measurement of dimethylxylindein in DCM (Figure 3) are displayed above to support the analysis and interpretation of excited-state structural dynamics. A longer time constant on the hundreds of ps timescale may be present due to triplet state formation; however, the vibrational peaks decay within the signal-to-noise ratio by $\sim 20$ ps (similar timescale to the ISC time constant, e.g., for $S_{1}$ or $S_{1}{ }^{\prime} \rightarrow T_{2}$ or $T_{2}$ ' transition in Figure 4), which indicates that the observed Raman peaks mostly track the singlet excited state dynamics (e.g., $\left.S_{1}\right)$. We note that these transient vibrational peaks do not display large frequency shifts, though the $1588 \mathrm{~cm}^{-1}$ peak red-shifts and the $1625 \mathrm{~cm}^{-1}$ peak blue-shifts by $\sim 3 \mathrm{~cm}^{-1}$, which could be attributed to the chromophore conformational change and vibrational cooling, respectively. ${ }^{25,36}$ The $1625 \mathrm{~cm}^{-1}$ mode consists of similar motions to the $1655 \mathrm{~cm}^{-1}$ mode (see Table S1) primarily involving the $\mathrm{C}=\mathrm{O}$ stretch (Figure S10a), while the pertinent motions of $1588 \mathrm{~cm}^{-1}$ peak are nearly identical to the $1551 \mathrm{~cm}^{-1}$ peak which involves $\mathrm{C}=\mathrm{C}$ stretch of the conjugated ring core (Figure $\mathrm{S} 10 \mathrm{~b}$ ).

In essence, the observed ES-FSRS peak dynamics infer that substituents toward the exterior of the dimethylxylindein molecular framework participate more during Franck-Condon relaxation, with a significantly larger amplitude weight of the $\sim 150$ fs initial decay component. In contrast, ring core motions are more active during $\mathrm{CT}$ relaxation on the $\sim 2 \mathrm{ps}$ timescale. We also attempted resonance enhancement with blue ESA band to track structural dynamics of triplet state; however, no strong peaks were observed. FSRS experimental conditions require careful optimization, as a Raman pump bluer than the actinic pump and GSA is not commonly performed because the Raman pump can act as a second excitation. ${ }^{44-46}$ Using Stokes FSRS with $480 \mathrm{~nm}$ Raman pump, the GSA can absorb Raman probe photons, complicating data collection; anti-Stokes FSRS is not possible given current experimental conditions and a lack of blue photons $(<480 \mathrm{~nm})$ for the Raman probe. 

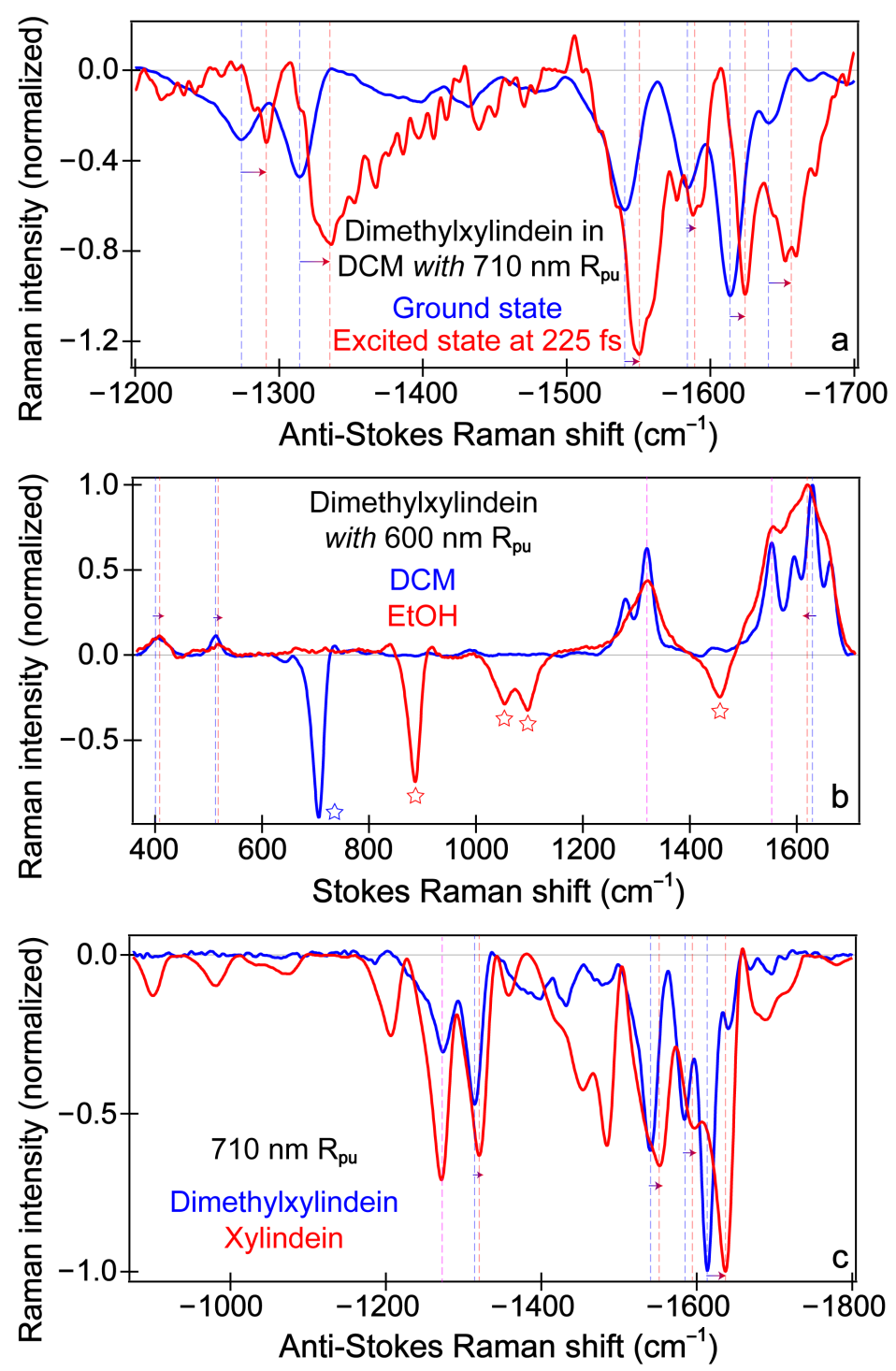

Figure S8. FSRS characterization of dimethylxylindein and xylindein. (a) Ground (blue) and excited-state (red) anti-Stokes FSRS spectra of dimethylxylindein in DCM. With a $710 \mathrm{~nm}$ Raman pump, the excited-state spectrum was taken at $225 \mathrm{fs}$ after an actinic pump at $560 \mathrm{~nm}$. (b) Groundstate Stokes FSRS spectra of dimethylxylindein in DCM (blue) and ethanol (red) with a $600 \mathrm{~nm}$ Raman pump. Color-coded stars denote dips due to solvent Raman modes. (c) Anti-Stokes groundstate FSRS spectra of dimethylxylindein (blue) and xylindein (red) in DCM with a $710 \mathrm{~nm}$ Raman pump. The anti-Stokes FSRS of xylindein resulted in positive peaks (Raman gain), ${ }^{47}$ the spectrum was therefore multiplied by -1 and displayed as negative peaks (Raman loss) to compare with the 
dimethylxylindein spectrum. Blue, red, and magenta vertical dashed lines highlight peak positions of the pertinent vibrational peaks, with arrows tracking pronounced frequency shifts. The spectra are normalized in each panel for a robust comparison.

Notably, the excited-state spectrum of dimethylxylindein in DCM at $225 \mathrm{fs}$ time delay shows a concerted blueshift relative to the ground-state peaks (Figure S8a). Many of these peaks involve motions of the carbonyl groups and conjugated core, supporting major assignment of the CT state formation upon photoexcitation (into Franck-Condon region). ${ }^{48,49}$ A few Raman modes, especially at 1655 and $1551 \mathrm{~cm}^{-1}$, become more intense in the excited state (Figure 3). In the more polar solvent ethanol, the enhanced H-bonding network red-shifts Raman peaks involving the $\mathrm{C}=\mathrm{O}$ stretch compared to DCM (Figure S8b); meanwhile, the motions involving the conjugated-ringcore $\mathrm{C}=\mathrm{C}$ motions show less of a frequency shift as they are less sensitive to the solvent. ${ }^{12}$

When comparing the GS-FSRS spectra of xylindein and dimethylxylindein in DCM (Figure S8c), many Raman peaks are conserved between two pigments, as expected given their similar chemical structures (Scheme 1). ${ }^{3,6}$ Several additional peaks are observed in the xylindein spectrum $\left(1688,1485 \mathrm{~cm}^{-1}\right)$ which have been assigned to motions involving the hydroxy group. In particular, the $1688 \mathrm{~cm}^{-1}$ mode involves localized $\mathrm{C}=\mathrm{O}$ stretching motions that are especially sensitive to the hydroxy group (i.e., a local probe).$^{18}$ The broadness of the $1688 \mathrm{~cm}^{-1}$ peak likely arises from two tautomers (see discussions following Figure $\mathrm{S} 1$ ), as the $\mathrm{C}=\mathrm{O}$ stretching frequencies are sensitive to local environment (heterogeneity) of the adjacent hydroxy group. Narrower Raman peaks of dimethylxylindein imply less conformational heterogeneity, while $710 \mathrm{~nm}$ Raman pump is more distant from its ground-state absorption peak vs. xylindein. ${ }^{12}$ The xylindein vibrational peaks are collectively blue-shifted from those of dimethylxylindein, implying a more conjugated electronic distribution throughout the molecule with stronger intramolecular H-bonding interactions. ${ }^{3,6}$ 


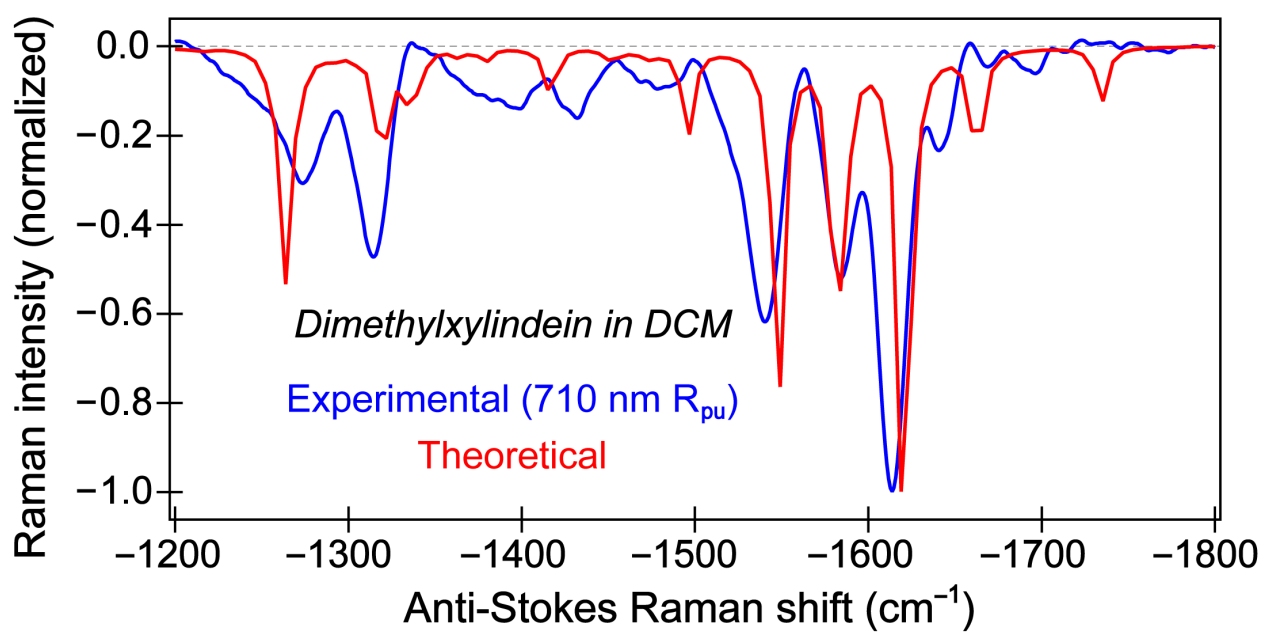

Figure S9. Comparison of the normalized dimethylxylindein ground-state experimental (blue) and theoretical (red) Raman spectra in DCM to aid mode assignment. The anti-Stokes FSRS spectrum was taken with a $710 \mathrm{~nm}$ Raman pump. Quantum calculations of the dimethylxylindein groundstate optimized structure in DCM (via IEFPCM to account for solvent effects) produced the theoretical spectrum with the full width at half maximum (FWHM) of $8 \mathrm{~cm}^{-1}$ for each normal mode. A frequency scaling factor of -0.99 (converting the Stokes to anti-Stokes Raman shift) and an intensity multiplication factor of -1 (converting the Raman gain to Raman loss) were applied to better match the theoretical and experimental spectra.

The theoretical and experimental Raman spectra of dimethylxylindein in DCM show good agreement. The experimental GS-FSRS peaks are broader than the calculated spectrum due to the ps duration of Raman pump (not continuous-wave Raman that achieves the natural linewidth) and the intrinsic chromophore heterogeneity in solution (which may not be accurately captured using the bulk solvent model without explicit solvent molecules) ${ }^{8,43}$ Furthermore, some differences in peak frequencies and relative intensities are expected due to the specific resonance enhancement conditions resulting in the experimental stimulated Raman gain (or loss), ${ }^{12}$ which is not accounted for in the off-resonance Raman theoretical spectrum plotted (see Experimental methods above). 


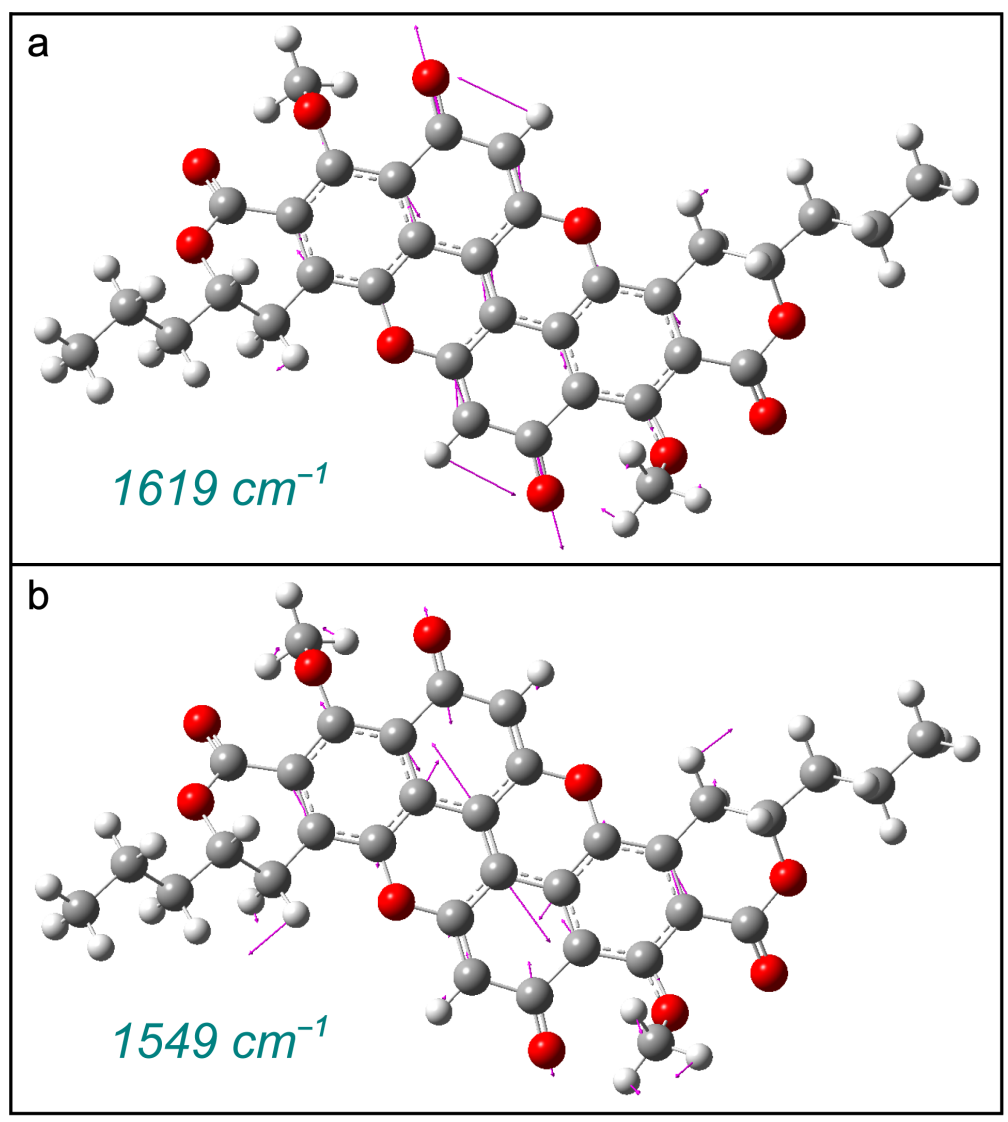

Figure S10. Illustrations for key vibrational modes of dimethylxylindein in solution. (a) The 1619 $\mathrm{cm}^{-1}$ and (b) $1549 \mathrm{~cm}^{-1}$ motions aided by quantum calculations with a frequency scaling factor of 0.99. The $\mathrm{C}, \mathrm{O}$, and $\mathrm{H}$ atoms are depicted as gray, red, and white spheres, respectively. Pertinent atomic motions of these calculated normal modes are shown as solid purple arrows. 


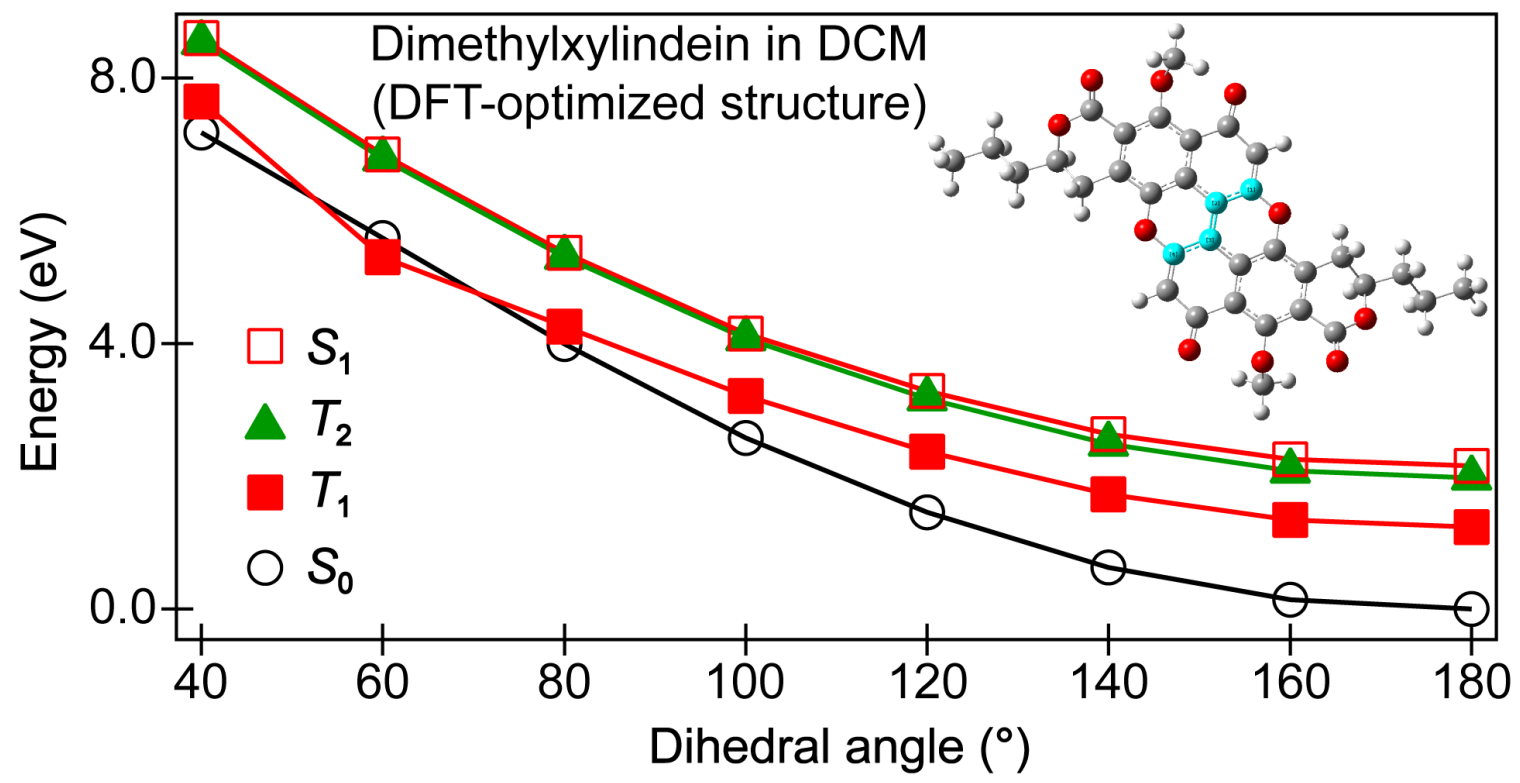

Figure S11. The coordinate-dependent potential energies of dimethylxylindein in DCM. Relative energies (in eV unit) of the electronic ground state $S_{0}$ (black circles) and the first several excited states $T_{1}$ (red squares), $T_{2}$ (green triangles), and $S_{1}$ (red hollow squares) were obtained from the DFT-optimized structures of dimethylxylindein in DCM solvent with the central dihedral angle (depicted by four carbon atoms in cyan in the inset) confined to discrete values from planar $\left(180^{\circ}\right)$ to non-planar $\left(\geq 40^{\circ}\right)$ conformations. The calculated energy trend was used to model the dimethylxylindein structural deviations from its conjugated-ring coplanarity that could promote ISC and/or internal conversion to a lower-lying state after electronic excitation. ${ }^{6}$ 


\section{$\underline{\text { SI Table }}$}

Table S1. Calculated Raman and experimental GS/ES-FSRS peak frequencies with vibrational normal mode assignment for dimethylxylindein in DCM

\begin{tabular}{|c|c|c|c|c|}
\hline $\begin{array}{l}\text { Calculated } \\
\text { frequency } \\
\left(\mathrm{cm}^{-1}\right)^{a}\end{array}$ & $\begin{array}{c}\text { GS peak } \\
\text { frequency } \\
\left(\mathrm{cm}^{-1}\right)^{b}\end{array}$ & $\begin{array}{c}\text { ES peak } \\
\text { frequency } \\
\left(\mathbf{c m}^{-1}\right)^{b}\end{array}$ & $\begin{array}{c}\text { GS } \rightarrow \text { ES peak } \\
\text { frequency blueshift } \\
\left(\mathrm{cm}^{-1}\right)\end{array}$ & $\begin{array}{c}\text { Vibrational } \\
\text { mode (major) } \\
\text { assignment }^{c}\end{array}$ \\
\hline 1660 & 1641 & 1655 & 14 & $\begin{array}{c}\mathrm{C}=\mathrm{O} \text { stretch, } \\
\text { weak } \mathrm{C}=\mathrm{C} \text { stretch }\end{array}$ \\
\hline $1619^{d}$ & 1614 & 1625 & 11 & $\begin{array}{c}\mathrm{C}=\mathrm{O} \text { stretch, } \\
\text { weak } \mathrm{C}=\mathrm{C} \text { stretch }\end{array}$ \\
\hline 1584 & 1584 & 1588 & 4 & $\begin{array}{c}\mathrm{C}=\mathrm{C} \text { stretch, } \\
\text { weak } \mathrm{C}=\mathrm{O} \text { stretch }\end{array}$ \\
\hline $1549^{d}$ & 1540 & 1551 & 11 & $\begin{array}{c}\mathrm{C}=\mathrm{C} \text { stretch, } \\
\text { weak } \mathrm{C}=\mathrm{O} \text { stretch }\end{array}$ \\
\hline 1322 & 1314 & 1335 & 21 & $\begin{array}{c}\mathrm{C}-\mathrm{H} \text { stretch, } \\
\text { weak } \mathrm{C}-\mathrm{C} \text { stretch }\end{array}$ \\
\hline 1264 & 1274 & 1290 & 16 & Ring breathing \\
\hline
\end{tabular}

${ }^{a}$ Ground-state (GS) Raman peak frequencies were calculated by DFT method using the B3LYP functional and 6-311+G(d,p) basis sets for the geometrically optimized chromophore structure. A frequency scaling of 0.99 was applied to better match the observed Raman peak frequencies. ${ }^{32,50}$

${ }^{b}$ GS and excited-state (ES) Raman peak frequencies were obtained from experimental GS- and ES-FSRS in solution with gaussian peak fitting to "filter" out spectral noise (Figure 3a). 7,8,12

${ }^{c}$ Raman mode assignments of the chromophore on the basis of major nuclear motions of calculated vibrational normal modes (see the dimethylxylindein chemical structure in Scheme 1, main text). ${ }^{d}$ Two key Raman marker bands (1619 and $1549 \mathrm{~cm}^{-1}$ in GS) with the corresponding ES peak intensity dynamics plots in Figure $\mathrm{S} 7\left(1625 \mathrm{~cm}^{-1}\right.$, blue trace $)$ and Figure $3 \mathrm{~b}\left(1551 \mathrm{~cm}^{-1}\right.$, red trace), as well as their associated nuclear motions illustrated in Figure S10a and S10b, respectively. 


\section{$\underline{\text { SI References }}$}

(1) Giesbers, G.; Van Schenck, J.; Vega Gutierrez, S.; Robinson, S.; Ostroverkhova, O. Fungi-Derived Pigments for Sustainable Organic (Opto)Electronics. MRS Adv. 2018, 3, 34593464.

(2) Giesbers, G.; Krueger, T.; Van Schenck, J.; Van Court, R.; Morré, J.; Fang, C.; Robinson, S.; Ostroverkhova, O. Fungi-Derived Xylindein: Effect of Purity on Optical and Electronic Properties. MRS Adv. 2019, 4, 1769-1777.

(3) Giesbers, G.; Krueger, T. D.; Van Schenck, J. D. B.; Kim, R.; Van Court, R. C.;

Robinson, S. C.; Beaudry, C. M.; Fang, C.; Ostroverkhova, O. Role of Hydroxyl Groups in the Photophysics, Photostability, and (Opto)electronic Properties of the Fungi-Derived Pigment Xylindein. J. Phys. Chem. C 2021, 125, 6534-6545.

(4) Giesbers, G.; Van Schenck, J.; Quinn, A.; Van Court, R.; Vega Gutierrez, S. M.;

Robinson, S. C.; Ostroverkhova, O. Xylindein: Naturally Produced Fungal Compound for Sustainable (Opto)electronics. ACS Omega 2019, 4, 13309-13318.

(5) Van Court, R. C.; Giesbers, G.; Ostroverkhova, O.; Robinson, S. C. Optimizing Xylindein from Chlorociboria spp. for (Opto)electronic Applications. Processes 2020, 8, 1477.

(6) Krueger, T. D.; Giesbers, G.; Van Court, R. C.; Zhu, L.; Kim, R.; Beaudry, C. M.; Robinson, S. C.; Ostroverkhova, O.; Fang, C. Ultrafast Dynamics and Photoresponse of a FungiDerived Pigment Xylindein from Solution to Thin Films. Chem. Eur. J. 2021, 27, 5627-5631.

(7) Liu, W.; Wang, Y.; Tang, L.; Oscar, B. G.; Zhu, L.; Fang, C. Panoramic Portrait of Primary Molecular Events Preceding Excited State Proton Transfer in Water. Chem. Sci. 2016, 7 , 5484-5494. 
(8) Fang, C.; Tang, L.; Oscar, B. G.; Chen, C. Capturing Structural Snapshots during Photochemical Reactions with Ultrafast Raman Spectroscopy: From Materials Transformation to Biosensor Responses. J. Phys. Chem. Lett. 2018, 9, 3253-3263.

(9) Tang, L.; Zhu, L.; Taylor, M. A.; Wang, Y.; Remington, S. J.; Fang, C. Excited State Structural Evolution of a GFP Single-Site Mutant Tracked by Tunable Femtosecond-Stimulated Raman Spectroscopy. Molecules 2018, 23, 2226.

(10) Zhu, L.; Liu, W.; Fang, C. A Versatile Femtosecond Stimulated Raman Spectroscopy Setup with Tunable Pulses in the Visible to Near Infrared. Appl. Phys. Lett. 2014, 105, 041106.

(11) Ostroverkhova, O. Organic Optoelectronic Materials: Mechanisms and Applications. Chem. Rev. 2016, 116, 13279-13412.

(12) Fang, C.; Tang, L.; Chen, C. Unveiling Coupled Electronic and Vibrational Motions of Chromophores in Condensed Phases. J. Chem. Phys. 2019, 151, 200901.

(13) Fang, C.; Frontiera, R. R.; Tran, R.; Mathies, R. A. Mapping GFP Structure Evolution During Proton Transfer with Femtosecond Raman Spectroscopy. Nature 2009, 462, 200-204.

(14) Frisch, M. J.; Trucks, G. W.; Schlegel, H. B.; Scuseria, G. E.; Robb, M. A.; Cheeseman, J. R.; Scalmani, G.; Barone, V.; Mennucci, B.; Petersson, G. A.; Nakatsuji, H.; Caricato, M.; Li, X.; Hratchian, H. P.; Izmaylov, A. F.; Bloino, J.; Zheng, G.; Sonnenberg, J. L.; Hada, M.; Ehara, M.; Toyota, K.; Fukuda, R.; Hasegawa, J.; Ishida, M.; Nakajima, T.; Honda, Y.; Kitao, O.; Nakai, H.; Vreven, T.; J. A. Montgomery, J.; Peralta, J. E.; Ogliaro, F.; Bearpark, M.; Heyd, J. J.; Brothers, E.; Kudin, K. N.; Staroverov, V. N.; Kobayashi, R.; Normand, J.; Raghavachari, K.; Rendell, A.; Burant, J. C.; Iyengar, S. S.; Tomasi, J.; Cossi, M.; Rega, N.; Millam, J. M.; Klene, M.; Knox, J. E.; Cross, J. B.; Bakken, V.; Adamo, C.; Jaramillo, J.; Gomperts, R.; Stratmann, R. E.; Yazyev, O.; Austin, A. J.; Cammi, R.; Pomelli, C.; Ochterski, J. W.; Martin, R. L.; Morokuma, K.; 
Zakrzewski, V. G.; Voth, G. A.; Salvador, P.; Dannenberg, J. J.; Dapprich, S.; Daniels, A. D.;

Farkas, Ö.; Foresman, J. B.; Ortiz, J. V.; Cioslowski, J.; Fox, D. J. Gaussian 09, Revision B.1;

Gaussian, Inc.: Wallingford, CT, 2009.

(15) Tang, L.; Fang, C. Nitration of Tyrosine Channels Photoenergy through a Conical Intersection in Water. J. Phys. Chem. B 2019, 123, 4915-4928.

(16) Aloïse, S.; Ruckebusch, C.; Blanchet, L.; Réhault, J.; Buntinx, G.; Huvenne, J.-P. The Benzophenone $\mathrm{S}_{1}\left(\mathrm{n}, \pi^{*}\right) \rightarrow \mathrm{T}_{1}\left(\mathrm{n}, \pi^{*}\right)$ States Intersystem Crossing Reinvestigated by Ultrafast Absorption Spectroscopy and Multivariate Curve Resolution. J. Phys. Chem. A 2008, 112, 224231.

(17) Zobel, J. P.; Nogueira, J. J.; González, L. Mechanism of Ultrafast Intersystem Crossing in 2-Nitronaphthalene. Chem. Eur. J. 2018, 24, 5379-5387.

(18) Hochstrasser, R. M. Two-Dimensional Spectroscopy at Infrared and Optical Frequencies. Proc. Natl. Acad. Sci. U. S. A. 2007, 104, 14190-14196.

(19) Reineke, S.; Baldo, M. A. Room Temperature Triplet State Spectroscopy of Organic Semiconductors. Sci. Rep. 2014, 4, 3797.

(20) Krueger, T. D.; Boulanger, S. A.; Zhu, L.; Tang, L.; Fang, C. Discovering a Rotational Barrier within a Charge-Transfer State of a Photoexcited Chromophore in Solution. Struct. Dyn. 2020, 7, 024901.

(21) Shao, Y.; Yang, Y. Efficient Organic Heterojunction Photovoltaic Cells Based on Triplet Materials. Adv. Mater. 2005, 17, 2841-2844.

(22) Sonoda, Y.; Kwok, W. M.; Petrasek, Z.; Ostler, R.; Matousek, P.; Towrie, M.; Parker, A. W.; Phillips, D. Solvent Effects on the Photophysical and Photochemical Properties of (E,E,E)1,6-bis(4-nitrophenyl)hexa-1,3,5-triene. J. Chem. Soc., Perkin Trans. 2 2001, 308-314. 
(23) Lv, M.; Yu, Y.; Sandoval-Salinas, M. E.; Xu, J.; Lei, Z.; Casanova, D.; Yang, Y.; Chen, J. Engineering the Charge-Transfer State to Facilitate Spin-Orbit Charge Transfer Intersystem Crossing in Spirobis[anthracene]diones. Angew. Chem. Int. Ed. 2020, 59, 22179-22184.

(24) Baba, M. Intersystem Crossing in the ${ }^{1} \mathrm{n} \pi^{*}$ and ${ }^{1} \pi \pi^{*}$ States. J. Phys. Chem. A 2011, 115, 9514-9519.

(25) Tang, L.; Wang, Y.; Zhu, L.; Lee, C.; Fang, C. Correlated Molecular Structural Motions for Photoprotection After Deep-UV Irradiation. J. Phys. Chem. Lett. 2018, 9, 2311-2319.

(26) van Stokkum, I. H. M.; Larsen, D. S.; van Grondelle, R. Global and Target Analysis of Time-Resolved Spectra. Biochim. Biophys. Acta 2004, 1657, 82-104.

(27) Snellenburg, J. J.; Laptenok, S. P.; Seger, R.; Mullen, K. M.; van Stokkum, I. H. M. Glotaran: A Java-Based Graphical User Interface for the R-Package TIMP. J. Stat. Softw. 2012, $49,1-22$.

(28) Shi, J.; Izquierdo, M. A.; Oh, S.; Park, S. Y.; Milián-Medina, B.; Roca-Sanjuán, D.; Gierschner, J. Inverted Energy Gap Law for the Nonradiative Decay in Fluorescent Floppy Molecules: Larger Fluorescence Quantum Yields for Smaller Energy Gaps. Org. Chem. Front. 2019, 6, 1948-1954.

(29) Chen, C.; Tachibana, S. R.; Baleeva, N. S.; Myasnyanko, I. N.; Bogdanov, A. M.; Gavrikov, A. S.; Mishin, A. S.; Malyshevskaya, K. K.; Baranov, M. S.; Fang, C. Developing Bright Green Fluorescent Protein (GFP)-like Fluorogens for Live-Cell Imaging with Nonpolar Protein-Chromophore Interactions. Chem. Eur. J. 2021, DOI: 10.1002/chem.202101250.

(30) Chen, C.; Liu, W.; Baranov, M. S.; Baleeva, N. S.; Yampolsky, I. V.; Zhu, L.; Wang, Y.; Shamir, A.; Solntsev, K. M.; Fang, C. Unveiling Structural Motions of a Highly Fluorescent 
Superphotoacid by Locking and Fluorinating the GFP Chromophore in Solution. J. Phys. Chem. Lett. 2017, 8, 5921-5928.

(31) Gai, F.; Fehr, M. J.; Petrich, J. W. Observation of Excited-State Tautomerization in the Antiviral Agent Hypericin and Identification of Its Fluorescent Species. J. Phys. Chem. 1994, 98, 5784-5795.

(32) Chen, C.; Zhu, L.; Baranov, M. S.; Tang, L.; Baleeva, N. S.; Smirnov, A. Y.; Yampolsky, I. V.; Solntsev, K. M.; Fang, C. Photoinduced Proton Transfer of GFP-Inspired Fluorescent Superphotoacids: Principles and Design. J. Phys. Chem. B 2019, 123, 3804-3821.

(33) Maroncelli, M.; Fleming, G. R. Picosecond Solvation Dynamics of Coumarin 153: The Importance of Molecular Aspects of Solvation. J. Chem. Phys. 1987, 86, 6221-6239.

(34) Baumler, S. M.; Mutchler, J. M.; Blanchard, G. J. Comparing Rotational and Translational Diffusion to Evaluate Heterogeneity in Binary Solvent Systems. J. Phys. Chem. B 2019, 123, 216-224.

(35) Tachibana, S. R.; Tang, L.; Chen, C.; Zhu, L.; Takeda, Y.; Fushimi, K.; Seevers, T. K.; Narikawa, R.; Sato, M.; Fang, C. Transient Electronic and Vibrational Signatures during Reversible Photoswitching of a Cyanobacteriochrome Photoreceptor. Spectrochim. Acta A 2021, $250,119379$.

(36) Tang, L.; Zhu, L.; Wang, Y.; Fang, C. Uncovering the Hidden Excited State toward Fluorescence of an Intracellular pH Indicator. J. Phys. Chem. Lett. 2018, 9, 4969-4975.

(37) Oscar, B. G.; Liu, W.; Rozanov, N. D.; Fang, C. Ultrafast Intermolecular Proton Transfer to a Proton Scavenger in an Organic Solvent. Phys. Chem. Chem. Phys. 2016, 18, 26151-26160. 
(38) Cui, G.; Fang, W.-h. State-Specific Heavy-Atom Effect on Intersystem Crossing Processes in 2-thiothymine: A Potential Photodynamic Therapy Photosensitizer. J. Chem. Phys. 2013, 138,044315 .

(39) Tilley, A. J.; Pensack, R. D.; Lee, T. S.; Djukic, B.; Scholes, G. D.; Seferos, D. S. Ultrafast Triplet Formation in Thionated Perylene Diimides. J. Phys. Chem. C 2014, 118, 999610004.

(40) El-Sayed, M. A. Spin-Orbit Coupling and the Radiationless Processes in Nitrogen Heterocyclics. J. Chem. Phys. 1963, 38, 2834-2838.

(41) Oberhofer, K. E.; Musheghyan, M.; Wegscheider, S.; Wörle, M.; Iglev, E. D.; Nikolova, R. D.; Kienberger, R.; Pekov, P. S.; Iglev, H. Individual Control of Singlet Lifetime and Triplet Yield in Halogen-Substituted Coumarin Derivatives. RSC Adv. 2020, 10, 27096-27102.

(42) Liu, W.; Tang, L.; Oscar, B. G.; Wang, Y.; Chen, C.; Fang, C. Tracking Ultrafast Vibrational Cooling During Excited State Proton Transfer Reaction with Anti-Stokes and Stokes Femtosecond Stimulated Raman Spectroscopy. J. Phys. Chem. Lett. 2017, 8, 997-1003.

(43) Dietze, D. R.; Mathies, R. A. Femtosecond Stimulated Raman Spectroscopy. ChemPhysChem 2016, 17, 1224-1251.

(44) Quick, M.; Dobryakov, A. L.; Kovalenko, S. A.; Ernsting, N. P. Resonance Femtosecond-Stimulated Raman Spectroscopy without Actinic Excitation Showing LowFrequency Vibrational Activity in the $\mathrm{S}_{2}$ State of All-Trans $\beta$-Carotene. J. Phys. Chem. Lett. 2015, $6,1216-1220$.

(45) Ferrante, C.; Pontecorvo, E.; Cerullo, G.; Vos, M. H.; Scopigno, T. Direct Observation of Subpicosecond Vibrational Dynamics in Photoexcited Myoglobin. Nat. Chem. 2016, 8, 11371143. 
(46) Fang, C.; Tang, L. Mapping Structural Dynamics of Proteins with Femtosecond Stimulated Raman Spectroscopy. Annu. Rev. Phys. Chem. 2020, 71, 239-265.

(47) Chen, C.; Zhu, L.; Fang, C. Femtosecond Stimulated Raman Line Shapes: Dependence on Resonance Conditions of Pump and Probe Pulses. Chin. J. Chem. Phys. 2018, 31, 492-502.

(48) Tominaga, K.; Walker, G. C.; Jarzeba, W.; Barbara, P. F. Ultrafast Charge Separation in ADMA: Experiment, Simulation, and Theoretical Issues. J. Phys. Chem. 1991, 95, 10475-10485.

(49) Taylor, M. A.; Zhu, L.; Rozanov, N. D.; Stout, K. T.; Chen, C.; Fang, C. Delayed Vibrational Modulation of the Solvated GFP Chromophore into a Conical Intersection. Phys. Chem. Chem. Phys. 2019, 21, 9728-9739.

(50) Merrick, J. P.; Moran, D.; Radom, L. An Evaluation of Harmonic Vibrational Frequency Scale Factors. J. Phys. Chem. A 2007, 111, 11683-11700. 